\title{
Invariant densities for random systems of the interval
}

\author{
CHARLENE KALLE and MARTA MAGGIONI \\ Mathematisch Instituut, Leiden University, Niels Bohrweg 1, \\ 2333CA Leiden, The Netherlands \\ (e-mail:kallecccj@math.leidenuniv.nl,m.maggioni@math.leidenuniv.nl)
}

(Received 5 July 2019 and accepted in revised form 2 October 2020)

\begin{abstract}
For random piecewise linear systems $T$ of the interval that are expanding on average we construct explicitly the density functions of absolutely continuous $T$-invariant measures. If the random system uses only expanding maps our procedure produces all invariant densities of the system. Examples include random tent maps, random $W$-shaped maps, random $\beta$-transformations and random Lüroth maps with a hole.
\end{abstract}

Key words: absolutely continuous invariant measure, $\beta$-expansions, interval map, Lüroth expansions, random dynamics

2020 Mathematics Subject Classification: 37E05, 28D05, 37H05 (Primary); 37A05, 37A44, 37A10, 60G10 (Secondary)

\section{Introduction}

The Perron-Frobenius operator has been used since the seminal paper [LY73] of Lasota and Yorke to establish the existence of absolutely continuous invariant measures for deterministic dynamical systems. The same approach was also successful in the study of random dynamical systems. In the random setting, instead of a single map, a family of maps is considered from which one is selected at each iteration at random. In [Pel84] Pelikan gave sufficient conditions under which a random system using a finite number of piecewise $C^{2}$-transformations on the interval has absolutely continuous invariant measures. He also discussed the possible number of ergodic components. Around the same time a similar result was obtained by Morita in [Mor85], allowing for the possibility to choose from an infinite family of maps as well. In more recent years these results have been generalized in various ways. See [Buz00, GB03, BG05, Ino12], for example.

Finding an explicit formula for the density functions of these absolutely continuous invariant measures, however, is a different matter. Here the Perron-Frobenius operator 
can only help if one can make an educated guess. An explicit expression for the invariant density is therefore available only for specific families of maps. In 1957 Rényi gave in [Rén57] an expression for the invariant density of the $\beta$-transformation $x \mapsto \beta x(\bmod 1)$ for $\beta=(1+\sqrt{5}) / 2$, the golden mean. Later Parry and Gel'fond gave a general formula for the invariant density of the $\beta$-transformation in [Par60, Gel59]. In [DK10] generalizations of the $\beta$-transformation were considered. A more general set-up allowing different slopes was proposed in [Kop90] by Kopf. He introduced a matrix $M$ for any piecewise linear expanding interval map satisfying some minor restraints and associated each absolutely continuous invariant measure of the system to a vector from the null space of $M$. Some twenty years later, Góra developed in [Gór09] a similar procedure for deterministic piecewise linear eventually expanding interval maps. Unless the map in question has many onto branches, the matrix involved in the procedure from [Gór09] is of higher dimension than the one used in [Kop90].

For random maps not much is known. An exception is the random $\beta$-transformation, which was first introduced in [DK03] by Dajani and Kraaikamp and uses random combinations of two piecewise linear maps with constant slope $\beta>1$. It has a unique absolutely continuous invariant measure, as proved in [DdV07]. In [Kem14] Kempton gave a formula for the invariant density in the case where one chooses the two base maps with equal probability. Recently Suzuki extended these results in [Suz19] to include the non-uniform Bernoulli regime as well.

This paper is concerned with finding explicit expressions for invariant densities of random systems. We consider any finite or countable family $\left\{T_{j}:[0,1] \rightarrow[0,1]\right\}$ of piecewise linear maps that are expanding on average. The random system $T$ is given by choosing one of these maps at each step using a probability vector $\mathbf{p}=\left(p_{j}\right)$. The existence of an absolutely continuous invariant measure for such systems is guaranteed by [Pel84] for a finite family and by [Buz00, Ino12] in the countable case. The main result of this paper is that we provide a procedure to construct explicit formulas for invariant probability densities of the random system $T$. This is the content of Theorem 4.1. The results from Theorem 4.1 cover those from [Kem14, Suz19] regarding the expression for the invariant density as a special case. If we assume that all maps $T_{j}$ are expanding, we obtain the stronger result that the procedure leading to Theorem 4.1 actually produces all absolutely continuous invariant measures of $T$. We prove this in Theorem 5.3.

The paper is organized as follows. In the second section we specify our set-up and introduce the necessary assumptions and notation. The third section is devoted to the definition of a matrix $M$ and to the proof that the null space of $M$ is non-trivial. In the fourth section we prove Theorem 4.1, relating each non-trivial vector $\gamma$ from the null space of $M$ to the density $h_{\gamma}$ of an absolutely continuous invariant measure of the system $T$. In the fifth section we prove Theorem 5.3 concerning when we get all invariant densities. It is in this section that the extra difficulties that we had to overcome for dealing with random systems instead of deterministic ones, are most visible. Finally, we apply the results to some examples. In the sixth section we consider random tent maps, random $W$-shaped maps and various random $\beta$-transformations. In the final section we elaborate on a system related to representations of real numbers: a random Lüroth map with a hole. 


\section{Preliminaries}

Let $\Omega \subseteq \mathbb{N}$ and let $\left\{T_{j}:[0,1] \rightarrow[0,1]\right\}_{j \in \Omega}$ be a family of piecewise linear transformations. Consider a positive probability vector $\mathbf{p}=\left(p_{j}\right)_{j \in \Omega}$ (i.e., $p_{j}>0$ for all $j \in \Omega$ and $\left.\sum_{j \in \Omega} p_{j}=1\right)$. We call the system $T$ a random system of the interval $[0,1]$ of probability $\mathbf{p}$, if for $x \in[0,1]$ and $j \in \Omega$,

$$
T(x):=T_{j}(x) \text { with probability } p_{j} .
$$

A measure $\mu_{\mathbf{p}}$ on $[0,1]$ is an absolutely continuous invariant measure for $T$ and $\mathbf{p}$ if there is a density function $h$, such that for each Borel set $A \subseteq[0,1]$ we have

$$
\mu_{\mathbf{p}}(A)=\int_{A} h d \lambda=\sum_{j \in \Omega} p_{j} \mu_{\mathbf{p}}\left(T_{j}^{-1} A\right),
$$

where $\lambda$ denotes the one-dimensional Lebesgue measure.

Such a random system $T$ can also be described by a pseudo skew-product system. In that case, let $\sigma: \Omega^{\mathbb{N}} \rightarrow \Omega^{\mathbb{N}}$ be the left shift on sequences and define the map $R: \Omega^{\mathbb{N}} \times$ $[0,1] \rightarrow \Omega^{\mathbb{N}} \times[0,1]$ by $R(\omega, x)=\left(\sigma(\omega), T_{\omega_{1}} x\right)$. If $m_{\mathbf{p}}$ is the p-Bernoulli measure on $\Omega^{\mathbb{N}}$, then $m_{\mathbf{p}} \times \mu_{\mathbf{p}}$ is an invariant measure for $R$. We call $R$ the pseudo skew-product system associated to $T$.

We put some assumptions on the systems $T$ we consider.

(A1) Assume that the set of all the critical points of the maps $T_{j}$ is finite. Denote these critical points by $0=z_{0}<z_{1}<\cdots<z_{N}=1$. The points $z_{i}$ together specify a common partition $\left\{I_{i}\right\}_{1 \leq i \leq N}$ of subintervals of $[0,1]$, such that all maps $T_{j}$ are monotone on each of the intervals $I_{i}$. Hence, there exist $k_{i, j}, d_{i, j} \in \mathbb{R}$ such that the maps $T_{i, j}:=\left.T_{j}\right|_{I_{i}}$ are given by

$$
T_{i, j}(x)=k_{i, j} x+d_{i, j}
$$

(A2) Assume that $T$ is expanding on average with respect to $\mathbf{p}$, that is, assume that there is a constant $0<\rho<1$, such that for all $x \in[0,1], \sum_{j \in \Omega}\left(p_{j} /\left|T_{j}^{\prime}(x)\right|\right) \leq \rho<1$. This is equivalent to assuming that for each $1 \leq i \leq N$,

$$
\sum_{j \in \Omega} \frac{p_{j}}{\left|k_{i, j}\right|} \leq \rho<1 .
$$

Under these conditions the random system $T$ satisfies conditions (a) and (b) from [Ino12], which studies the existence of invariant densities $h$ satisfying (1) using the Perron-Frobenius operator. For the deterministic maps $T_{j}:[0,1] \rightarrow[0,1], j \in \Omega$, the Perron-Frobenius operator on $L^{1}(\lambda)$ is given by

$$
P_{T_{j}} f(x)=\sum_{y \in T_{j}^{-1}\{x\}} \frac{f(y)}{\left|T_{j}^{\prime}(y)\right|} .
$$

The random Perron-Frobenius operator is then defined by

$$
P_{T} f=\sum_{j \in \Omega} p_{j} P_{T_{j}} f
$$


The operator $P_{T}$ is clearly linear and positive. An $L^{1}(\lambda)$-function $h$ is called $T$-invariant for the random system $T$ if it is a fixed point of $P_{T}$, that is, if it satisfies $P_{T} h=h \lambda$-almost everywhere (a.e.). A density function $h$ is the density of a measure $\mu_{\mathbf{p}}$ satisfying (1) if and only if it is a fixed point of $P_{T}$. From [Ino12, Theorem 5.2] it follows that a $T$-invariant measure $\mu_{\mathbf{p}}$ of the form (1), and hence a $T$-invariant function $h$, exists. Inoue obtained this result by showing that the operator $P_{T}$, applied to functions of bounded variation, satisfies a Lasota-Yorke type inequality. From the famous Ionescu-Tulcea and Marinescu theorem one can then deduce much more than mere existence of an absolutely continuous invariant measure; it says that $P_{T}$ as an operator on the space of functions of bounded variation is quasi-compact. The specific implications of the quasi-compactness of $P_{T}$ that we use in this paper are the following. The eigenvalue 1 of $P_{T}$ has a finite-dimensional eigenspace. In other words, the subspace of $L^{1}(\lambda)$ of $T$-invariant functions is a finite-dimensional sublattice of the space of functions of bounded variation. As such, it has a finite base $H=$ $\left\{v_{1}, \ldots, v_{r}\right\}$ of $T$-invariant density functions of bounded variation, each corresponding to an ergodic measure, so that any other $T$-invariant $L^{1}(\lambda)$-function $h$ can be written as a linear combination of the $v_{i}: h=\sum_{i=1}^{r} c_{i} v_{i}$ for some constants $c_{i} \in \mathbb{R}$. Furthermore, if we set $U_{i}:=\left\{x: v_{i}(x)>0\right\}$ for the support of the function $v_{i}$, then each $U_{i}$ is forward invariant under $T$ in the sense that

$$
\lambda\left(U_{i} \triangle \bigcup_{j \in \Omega} T_{j}\left(U_{i}\right)\right)=0,
$$

where $\triangle$ denotes the symmetric difference. Also, the sets $U_{i}$ are mutually disjoint and none of the sets $U_{i}$ can properly contain another forward invariant set. We will use these properties in the proofs from $\S 5$. An account of these implications on the operator $P_{T}$ can be found in [Pel84, Mor85, Buz00, Ino12], for example. For more information, we also refer to standard textbooks such as [BG97, LM94].

In this paper we find $T$-invariant functions $h:[0,1] \rightarrow \mathbb{R}$ by linking them to the vectors from the null space of a matrix $M$. To guarantee that this null space is non-trivial, we formulate three additional assumptions that are easy to verify for any given systems. Firstly, we assume that not all the lines $x \mapsto k_{i, j} x+d_{i, j}, 1 \leq i \leq N$, with respective weights $p_{j}$, have a common intersection point with the diagonal. More precisely, consider for each interval $I_{i}$ the weighted intersection point with the diagonal

$$
x=\sum_{j \in \Omega} p_{j}\left(\frac{x}{k_{i, j}}-\frac{d_{i, j}}{k_{i, j}}\right) .
$$

Our third assumption states that for each $i$ there is an $n$, such that these points do not coincide.

(A3) Assume that for each $1 \leq i \leq N$, there is an $1 \leq n \leq N$, such that

$$
\frac{\sum_{j \in \Omega}\left(p_{j} / k_{i, j}\right) d_{i, j}}{1-\sum_{j \in \Omega}\left(p_{j} / k_{i, j}\right)} \neq \frac{\sum_{j \in \Omega}\left(p_{j} / k_{n, j}\right) d_{n, j}}{1-\sum_{j \in \Omega}\left(p_{j} / k_{n, j}\right)} .
$$



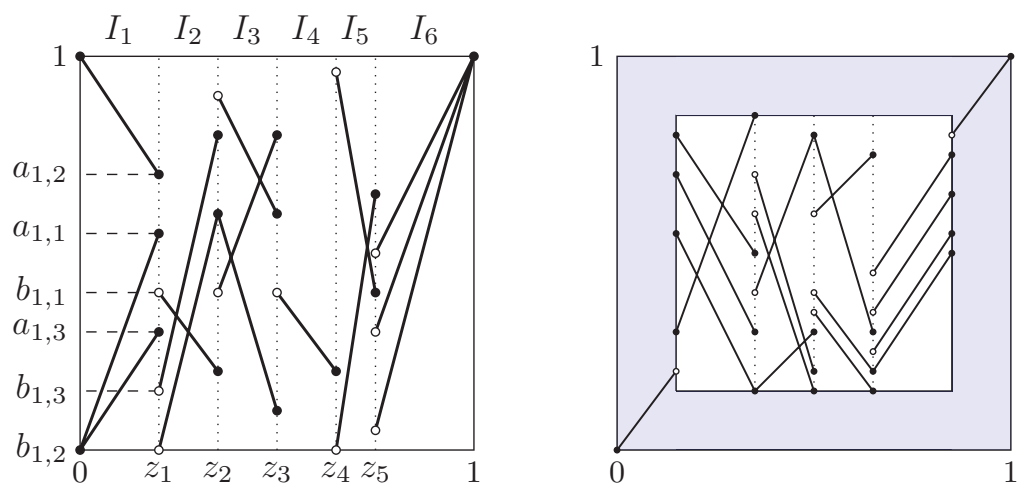

FIGURE 1 . On the left is an arbitrary map $T$ satisfying the above conditions. On the right we see a random map $T$ in the white box that does not satisfy (A4). By adding the branches in the grey part and rescaling, we obtain a system that does satisfy these conditions. Note that any point in the grey part (except for 0 and 1) moves to the white part after a finite number of iterations and stays there. Hence, any invariant density will equal 0 on the grey part.

Note that if $d_{i, j}<0$, then $k_{i, j}>-d_{i, j}$ and if $d_{i, j}>1$, then $k_{i, j}<1-d_{i, j}$. Hence, in all cases $\left|d_{i, j}\right|<\left|k_{i, j}\right|+1$ and by (A2),

$$
\sum_{j \in \Omega} \frac{p_{j}}{\left|k_{i, j}\right|}\left|d_{i, j}\right| \leq 1+\rho .
$$

So, the quantities in (A3) are all finite. Our fourth assumption is on the orbits of the points 0 and 1.

(A4) For each $j$, assume that

$$
d_{1, j}=\left\{\begin{array}{ll}
0 & \text { if } k_{1, j}>0, \\
1 & \text { if } k_{1, j}<0,
\end{array} \quad \text { and } \quad d_{N, j}= \begin{cases}1-k_{N, j} & \text { if } k_{N, j}>0, \\
-k_{N, j} & \text { if } k_{N, j}<0 .\end{cases}\right.
$$

In other words, the points 0 and 1 are mapped to 0 or 1 under all maps $T_{j}$, making the system continuous at the origin, when we consider it as acting on the circle $\mathbb{R} / \mathbb{Z}$ with the points 0 and 1 identified. Since we can deal with finitely many discontinuities, (A4) is not necessary for our results to hold, but it makes computations easier. Any system not satisfying it can be extended to a system that does satisfy this condition and for which no absolutely continuous invariant measure puts weight on the added pieces. See Figure 1 for an illustration and see $\S 6.3$ for a concrete example, given by the random $(\alpha, \beta)$-transformation.

Finally, we include an assumption stating that the weighted inverse derivative cannot be 0 anywhere.

(A5) Assume that for any $x \in[0,1]$, the weighted inverse derivative satisfies $\sum_{j \in \Omega}\left(p_{j} / T_{j}^{\prime}(x)\right) \neq 0$. This is equivalent to assuming that for each $1 \leq i \leq N$,

$$
\sum_{j \in \Omega} \frac{p_{j}}{k_{i, j}} \neq 0
$$


Conditions (A3) and (A5) are sufficient to get our main results, but probably not necessary. Note that (A5) is automatically fulfilled for any deterministic Lasota-Yorke map (and, in particular, for any deterministic piecewise linear map) and also for any random system for which on each interval $I_{i}$ the derivatives of all maps $T_{j}$ have the same sign. The final section contains an example that does not satisfy (A5) for a specific choice of $\mathbf{p}$. We will see that the procedure which leads to our main results still gives all invariant densities in that case. Moreover, if (A5) is not satisfied for some probability vector $\mathbf{p}$, then changing p slightly already lifts this restriction.

\section{A homogeneous system with a non-trivial solution}

An invariant measure reflects the dynamics of a system. For the maps $T_{j}, j \in \Omega$, considered in this article, the dynamics is determined by the orbits of the endpoints of the lines $x \mapsto k_{i, j} x+d_{i, j}, 1 \leq i \leq N$. We start this section by defining some quantities that keep track of the possible orbits of these points.

Let $\Omega^{*}$ be the set of all finite strings of elements from $\Omega$ together with the empty string $\varepsilon$. For $t \geq 0$, let $\Omega^{t} \subseteq \Omega^{*}$ denote the subset of those strings that have length $t$. So, in particular, $\Omega^{0}=\{\varepsilon\}$. Let $|\omega|$ denote the length of the string $\omega$. For any string $\omega \in \Omega^{*}$ with $|\omega| \geq t$, we let $\omega_{1}^{t}$ denote the starting block of length $t$. For two strings $\omega, \omega^{\prime} \in \Omega^{*}$ we simply write $\omega \omega^{\prime}$ for their concatenation. Each element $\omega \in \Omega^{t}$ defines a possible start of an orbit of a point in $[0,1]$ by composition of maps: for $x \in[0,1]$ and $\omega=\omega_{1} \cdots \omega_{t} \in \Omega^{t}$, define

$$
T_{\omega}(x)=T_{\omega_{t}} \circ T_{\omega_{t-1}} \circ \cdots \circ T_{\omega_{1}}(x)
$$

and set $T_{\varepsilon}(x)=x$. For $\omega \in \Omega^{*}$, set $\tau_{\omega}(y, 0)=1$, and for $1 \leq t \leq|\omega|$, set

$$
\tau_{\omega}(y, t):=\frac{p_{\omega_{t}}}{k_{i, \omega_{t}}} \quad \text { if } T_{\omega_{1}^{t-1}}(y) \in I_{i} .
$$

Define

$$
\delta_{\omega}(y, t):=\prod_{n=0}^{t} \tau_{\omega}(y, n),
$$

so that $\delta_{\omega}(y, t)$ is the weighted slope of the map $T_{\omega_{1}^{t}}$ at the point $y$. Note that $\tau_{\omega}(y, t)$ and $\delta_{\omega}(y, t)$ only depend on the block $\omega_{1}^{t}$ and not on what comes after. Moreover, for a concatenation $\omega j$, given by any block $\omega$ with $|\omega|=t-1$ and any $j \in \Omega$, we have that $\tau_{\omega j}(y, t)=\tau_{j}\left(T_{\omega}(y), 1\right)$ and $\delta_{\omega j}(y, t)=\tau_{\omega j}(y, t) \delta_{\omega}(y, t-1)$. By assumption (A2) we have that for any $y \in[0,1]$,

$$
\begin{aligned}
\left|\sum_{t \geq 0} \sum_{\omega \in \Omega^{t}} \delta_{\omega}(y, t)\right| & \leq 1+\sum_{t \geq 1} \sum_{\omega \in \Omega^{t-1}} \sum_{j \in \Omega}\left|\delta_{\omega}(y, t-1)\right|\left|\tau_{\omega j}(y, t)\right| \\
& \leq 1+\sum_{t \geq 1} \sum_{\omega \in \Omega^{t-1}}\left|\delta_{\omega}(y, t-1)\right| \rho \leq \frac{1}{1-\rho} .
\end{aligned}
$$

Let $\mathbf{1}_{A}$ denote the characteristic function of the set $A$ and set

$$
\mathrm{KI}_{n}(y):=\sum_{t \geq 1} \sum_{\omega \in \Omega^{t}} \delta_{\omega}(y, t) \mathbf{1}_{I_{n}}\left(T_{\omega_{1}^{t-1}}(y)\right) \quad \text { for } 1 \leq n \leq N .
$$


Then $\mathrm{KI}_{n}(y)$ keeps track of all the times the random orbit of $y$ visits $I_{n}$ and adds the corresponding weighted slopes. For $1 \leq i \leq N-1$, set $A_{i}:=I_{1} \cup \cdots \cup I_{i}$ and $B_{i}:=$ $I_{i+1} \cup \cdots \cup I_{N}$. We define

$$
\begin{aligned}
\mathrm{KA}_{i}(y) & :=\sum_{t \geq 0} \sum_{\omega \in \Omega^{t}} \delta_{\omega}(y, t) \mathbf{1}_{A_{i}}\left(T_{\omega}(y)\right), \\
\mathrm{KB}_{i}(y) & :=\sum_{t \geq 0} \sum_{\omega \in \Omega^{t}} \delta_{\omega}(y, t) \mathbf{1}_{B_{i}}\left(T_{\omega}(y)\right) .
\end{aligned}
$$

By (6), $\left|\mathrm{KI}_{n}\right|,\left|\mathrm{KA}_{i}\right|$ and $\left|\mathrm{KB}_{i}\right|$ are finite for all $y \in[0,1]$. For each $1 \leq n \leq N$, let $S_{n}$ be the average inverse of the slope,

$$
S_{n}:=\sum_{j \in \Omega} \frac{p_{j}}{k_{n, j}}
$$

which is non-zero by (A5), so that $S_{n}^{-1}$ is well defined. The next two lemmas give some identities that we will use later.

LEMMA 3.1. For each $y \in[0,1]$ and $1 \leq i \leq N-1$ we have

$$
\mathrm{KA}_{i}(y)=\sum_{n=1}^{i} S_{n}^{-1} \mathrm{KI}_{n}(y) \text { and } \mathrm{KB}_{i}(y)=\sum_{n=i+1}^{N} S_{n}^{-1} \mathrm{KI}_{n}(y) \text {. }
$$

Proof. For any $1 \leq n \leq N$ we have

$$
\begin{aligned}
\sum_{t \geq 0} \sum_{\omega \in \Omega^{t}} \delta_{\omega}(y, t) \mathbf{1}_{I_{n}}\left(T_{\omega}(y)\right) & =\sum_{t \geq 0} \sum_{\omega \in \Omega^{t}}\left(\sum_{j \in \Omega} \frac{p_{j}}{k_{n, j}}\right)^{-1}\left(\sum_{j \in \Omega} \frac{p_{j}}{k_{n, j}}\right) \delta_{\omega}(y, t) \mathbf{1}_{I_{n}}\left(T_{\omega}(y)\right) \\
& =\sum_{t \geq 0} \sum_{\omega \in \Omega^{t}} S_{n}^{-1} \sum_{j \in \Omega} \tau_{\omega j}(y, t+1) \delta_{\omega}(y, t) \mathbf{1}_{I_{n}}\left(T_{\omega}(y)\right) \\
& =S_{n}^{-1} \sum_{t \geq 0} \sum_{\omega \in \Omega^{t+1}} \delta_{\omega}(y, t+1) \mathbf{1}_{I_{n}}\left(T_{\omega_{1}^{t}}(y)\right)=S_{n}^{-1} \operatorname{KI}_{n}(y) .
\end{aligned}
$$

Putting this in the definition of $\mathrm{KA}_{i}(y)$ from (7) gives the first part of the lemma. Using (8), we also get that

$$
\mathrm{KA}_{i}(y)+\mathrm{KB}_{i}(y)=\sum_{t \geq 0} \sum_{\omega \in \Omega^{t}} \delta_{\omega}(y, t)=\sum_{n=1}^{N} S_{n}^{-1} \mathrm{KI}_{n}(y) .
$$

The result for $\mathrm{KB}_{i}$ follows.

Define

$$
K_{n}:=S_{n}^{-1}-1 \quad \text { and } \quad D_{n}:=S_{n}^{-1}\left(\sum_{j \in \Omega} \frac{p_{j}}{k_{n, j}} d_{n, j}\right)
$$

Then

$$
\frac{D_{n}}{K_{n}}=\frac{\sum_{j \in \Omega}\left(p_{j} / k_{n, j}\right) d_{n, j}}{1-\sum_{j \in \Omega}\left(p_{j} / k_{n, j}\right)}
$$


so that we can rephrase assumption (A3) as follows: for each $1 \leq i \leq N$, there is an $1 \leq$ $n \leq N$ such that $D_{i} / K_{i} \neq D_{n} / K_{n}$. We have the following properties for $K_{n}$ and $D_{n}$.

LEMMA 3.2. Let $y \in[0,1]$. Then

$$
\sum_{n=1}^{N} K_{n} \mathrm{KI}_{n}(y)=1 \quad \text { and } \quad-\sum_{n=1}^{N} D_{n} \mathrm{KI}_{n}(y)=y .
$$

Proof. For the first part, note that by (9) we have

$$
\sum_{n=1}^{N} S_{n}^{-1} \mathrm{KI}_{n}(y)=1+\sum_{t \geq 1} \sum_{\omega \in \Omega^{t}} \delta_{\omega}(y, t)=1+\sum_{n=1}^{N} \mathrm{KI}_{n}(y) .
$$

For the second part, let $1 \leq i \leq N$ be such that $y \in I_{i}$. Then for $j \in \Omega$ we get $T_{i, j}(y)=$ $k_{i, j} y+d_{i, j}$, and thus

$$
y=\sum_{j \in \Omega}\left(\frac{p_{j}}{k_{i, j}} T_{i, j}(y)-\frac{p_{j}}{k_{i, j}} d_{i, j}\right) .
$$

For $t \geq 1$ and $\omega \in \Omega^{*}$ with $|\omega| \geq t$, set

$$
\theta_{\omega}(y, t):=-\frac{p_{\omega_{t}}}{k_{n, \omega_{t}}} d_{n, \omega_{t}} \quad \text { if } T_{\omega_{1}^{t-1}}(y) \in I_{n} .
$$

Then

$$
y=\sum_{\omega \in \Omega} \tau_{\omega}(y, 1) T_{\omega}(y)+\theta_{\omega}(y, 1) .
$$

Since $\tau_{j}\left(T_{\omega}(y), 1\right)=\tau_{\omega j}(y, 2)$ and $\theta_{j}\left(T_{\omega}(y), 1\right)=\theta_{\omega j}(y, 2)$, we obtain for $\omega \in \Omega$ that

$$
T_{\omega}(y)=\sum_{j \in \Omega} \tau_{\omega j}(y, 2) T_{\omega j}(y)+\theta_{\omega j}(y, 2)
$$

Repeated application of (13) in (12), together with the definition of $\delta_{\omega}$ from (5), yields after $n$ steps,

$$
y=\sum_{t=1}^{n+1} \sum_{\omega \in \Omega^{t}} \delta_{\omega}(y, t-1) \theta_{\omega}(y, t)+\sum_{\omega \in \Omega^{n+1}} \delta_{\omega}(y, n+1) T_{\omega}(y) .
$$

From (6) we obtain that $\lim _{n \rightarrow \infty} \sum_{\omega \in \Omega^{n+1}}\left|\delta_{\omega}(y, n+1) T_{\omega}(y)\right|=0$. Hence, by (A2), (4) and (6),

$$
\begin{aligned}
y & =\sum_{t \geq 0} \sum_{\omega \in \Omega^{t+1}} \delta_{\omega}(y, t) \theta_{\omega}(y, t+1) \\
& =-\sum_{n=1}^{N} \sum_{t \geq 0} \sum_{\omega \in \Omega^{t}} \delta_{\omega}(y, t) \mathbf{1}_{I_{n}}\left(T_{\omega}(y)\right)\left(\sum_{j \in \Omega} \frac{p_{j}}{k_{n, j}} d_{n, j}\right)
\end{aligned}
$$




$$
\begin{aligned}
& =-\sum_{n=1}^{N} S_{n}^{-1}\left(\sum_{j \in \Omega} \frac{p_{j}}{k_{n, j}} d_{n, j}\right) \sum_{t \geq 0} \sum_{\omega \in \Omega^{t}} \delta_{\omega}(y, t)\left(\sum_{j \in \Omega} \frac{p_{j}}{k_{n, j}}\right) \mathbf{1}_{I_{n}}\left(T_{\omega}(y)\right) \\
& =-\sum_{n=1}^{N} D_{n} \sum_{t \geq 0} \sum_{\omega \in \Omega^{t}} \delta_{\omega}(y, t)\left(\sum_{j \in \Omega} \tau_{\omega j}(y, t+1)\right) \mathbf{1}_{I_{n}}\left(T_{\omega}(y)\right) \\
& =-\sum_{n=1}^{N} D_{n} \sum_{t \geq 0} \sum_{\omega \in \Omega^{t+1}} \delta_{\omega}(y, t+1) \mathbf{1}_{I_{n}}\left(T_{\omega_{1}^{t}}(y)\right)=-\sum_{n=1}^{N} D_{n} \mathrm{KI}_{n}(y) .
\end{aligned}
$$

For the invariant densities, we need to keep track of the orbits of the limits from the left and from the right of each partition point. Set, for $1 \leq i \leq N-1$ and $j \in \Omega$,

$$
a_{i, j}:=k_{i, j} z_{i}+d_{i, j}=\lim _{x \uparrow z_{i}} T_{j}(x), \quad \text { and } \quad b_{i, j}:=k_{i+1, j} z_{i}+d_{i+1, j}=\lim _{x \downarrow z_{i}} T_{j}(x) .
$$

See also Figure 1.

Definition 3.3. The $N \times(N-1)$ matrix $M=\left(\mu_{n, i}\right)$ given by

$$
\mu_{n, i}:= \begin{cases}\sum_{j \in \Omega}\left[\frac{p_{j}}{k_{i, j}}+\frac{p_{j}}{k_{i, j}} \mathrm{KI}_{n}\left(a_{i, j}\right)-\frac{p_{j}}{k_{i+1, j}} \mathrm{KI}_{n}\left(b_{i, j}\right)\right] & \text { for } n=i, \\ \sum_{j \in \Omega}\left[\frac{p_{j}}{k_{i, j}} \mathrm{KI}_{n}\left(a_{i, j}\right)-\frac{p_{j}}{k_{i+1, j}}-\frac{p_{j}}{k_{i+1, j}} \mathrm{KI}_{n}\left(b_{i, j}\right)\right] & \text { for } n=i+1, \\ \sum_{j \in \Omega}\left[\frac{p_{j}}{k_{i, j}} \mathrm{KI}_{n}\left(a_{i, j}\right)-\frac{p_{j}}{k_{i+1, j}} \mathrm{KI}_{n}\left(b_{i, j}\right)\right] & \text { otherwise, }\end{cases}
$$

is called the fundamental matrix of the random piecewise linear system $T$.

Note that assumption (A2) together with the fact that $\left|\mathrm{KI}_{n}(y)\right|<\infty$ for all $y \in[0,1]$ implies that all entries of $M$ are finite. In the next section we associate invariant functions $h_{\gamma}$ to vectors $\gamma \in \mathbb{R}^{N-1}$ in the null space of $M$. Here we prove that the null space of $M$ is non-trivial.

LEMMA 3.4. The system $M \gamma=0$ admits at least one non-trivial solution.

Proof. Since $M$ has dimension $N \times(N-1)$, by the Rouché-Capelli theorem the associated homogeneous system admits a non-trivial solution if and only if the rank of $M$ is at most $N-2$. Below we will give non-trivial linear dependence relations between all combinations of $N-1$ out of $N$ rows. It follows that any minor of order $N-1$ of $M$ is zero and thus that the rank of $M$ is at most $N-2$. We first show that for every $1 \leq i \leq N-1$,

$$
\sum_{n=1}^{N} K_{n} \mu_{n, i}=0 \quad \text { and } \quad \sum_{n=1}^{N} D_{n} \mu_{n, i}=0 .
$$


Indeed by Lemma 3.2,

$$
\begin{aligned}
& \sum_{n=1}^{N} K_{n} \mu_{n, i} \\
& \quad=\sum_{j \in \Omega}\left[\frac{p_{j}}{k_{i, j}} K_{i}-\frac{p_{j}}{k_{i+1, j}} K_{i+1}+\frac{p_{j}}{k_{i, j}} \sum_{n=1}^{N} K_{n} \mathrm{KI}_{n}\left(a_{i, j}\right)-\frac{p_{j}}{k_{i+1, j}} \sum_{n=1}^{N} K_{n} \mathrm{KI}_{n}\left(b_{i, j}\right)\right] \\
& \quad=S_{i}\left(S_{i}^{-1}-1\right)-S_{i+1}\left(S_{i+1}^{-1}-1\right)+S_{i}-S_{i+1}=0 .
\end{aligned}
$$

On the other hand, by the definition of the points $a_{i, j}$ and $b_{i, j}$,

$$
\begin{aligned}
& \sum_{n=1}^{N} D_{n} \mu_{n, i} \\
& \quad=\sum_{j \in \Omega}\left[\frac{p_{j}}{k_{i, j}} D_{i}-\frac{p_{j}}{k_{i+1, j}} D_{i+1}+\frac{p_{j}}{k_{i, j}} \sum_{n=1}^{N} D_{n} \mathrm{KI}_{n}\left(a_{i, j}\right)-\frac{p_{j}}{k_{i+1, j}} \sum_{n=1}^{N} D_{n} \mathrm{KI}_{n}\left(b_{i, j}\right)\right] \\
& \quad=\sum_{j \in \Omega}\left(S_{i} S_{i}^{-1} \frac{p_{j}}{k_{i, j}} d_{i, j}-S_{i+1} S_{i+1}^{-1} \frac{p_{j}}{k_{i+1, j}} d_{i+1, j}-\frac{p_{j}}{k_{i, j}} a_{i, j}+\frac{p_{j}}{k_{i+1, j}} b_{i, j}\right)=0 .
\end{aligned}
$$

Consequently, for every $1 \leq l \leq N$ and every $1 \leq i \leq N-1$,

$$
\sum_{n=1, n \neq l}^{N}\left(D_{l} K_{n}-D_{n} K_{l}\right) \mu_{n, i}=0 .
$$

By assumption (A3) this gives non-trivial linear dependence relations between all combinations of $N-1$ out of $N$ rows, giving the result.

Remark 3.5. Note that if $S_{n}=0$ for some $1 \leq n \leq N$, then the quantities $K_{n}$ and $D_{n}$ are not well defined. In this case $\mu_{n, i}=\sum_{j \in \Omega}\left(p_{j} / k_{i, j}\right) \mathrm{KI}_{n}\left(a_{i, j}\right)-\left(p_{j} /\left(k_{i+1, j}\right)\right) \mathrm{KI}_{n}\left(b_{i, j}\right)$ for each $1 \leq i \leq N-1$ and by the definition of $\mathrm{KI}_{n}$ we can write for any $y \in[0,1]$ that

$$
\begin{aligned}
\mathrm{KI}_{n}(y) & =\sum_{t \geq 1} \sum_{\omega \in \Omega^{t-1}} \sum_{j \in \Omega} \delta_{\omega}(y, t-1) \frac{p_{j}}{k_{n, j}} 1_{I_{n}}\left(T_{\omega_{1}^{t-1}}(y)\right) \\
& =\sum_{t \geq 1} \sum_{\omega \in \Omega^{t-1}} \delta_{\omega}(y, t-1) 1_{I_{n}}\left(T_{\omega_{1}^{t-1}}(y)\right) S_{n}=0 .
\end{aligned}
$$

Hence, $\mu_{n, i}=0$ for each $i$. From this, it is clear that if $S_{n}=0$ for at least two indices $n$, then a non-trivial vector $\gamma$ such that $M \gamma=0$ still exists. If there is a unique $\ell$ with $S_{\ell}=0$, then to obtain a non-trivial solution one still needs to find suitable constants $c_{n}$ such that $\sum_{n=1, n \neq \ell}^{N} c_{n} \mu_{n, i}=0$ for each $i$.

Any vector $\gamma$ from the null space of $M$ satisfies the following orthogonal relations, linking $\gamma$ to the functions $\mathrm{KA}_{i}$ and $\mathrm{KB}_{i}$. 
LEMMA 3.6. For all $1 \leq i \leq N-1$ we have the following orthogonal relations:

$$
\gamma_{i}+\sum_{m=1}^{N-1} \gamma_{m} \sum_{j \in \Omega}\left[\frac{p_{j}}{k_{m, j}} \mathrm{KA}_{i}\left(a_{m, j}\right)-\frac{p_{j}}{k_{m+1, j}} \mathrm{KA}_{i}\left(b_{m, j}\right)\right]=0,
$$

and

$$
\gamma_{i}-\sum_{m=1}^{N-1} \gamma_{m} \sum_{j \in \Omega}\left[\frac{p_{j}}{k_{m, j}} \mathrm{~KB}_{i}\left(a_{m, j}\right)-\frac{p_{j}}{k_{m+1, j}} \mathrm{~KB}_{i}\left(b_{m, j}\right)\right]=0 .
$$

Proof. If $\gamma$ is a solution of the system $M \gamma=0$, then $\sum_{m=1}^{N-1} \gamma_{m} \mu_{n, m}=0$ for all $n$. Lemma 3.1 gives for $n=1$,

$$
\begin{aligned}
0 & =S_{1}^{-1} \sum_{m=1}^{N-1} \gamma_{m} \mu_{1, m} \\
& =S_{1}^{-1} \gamma_{1} \sum_{j \in \Omega} \frac{p_{j}}{k_{1, j}}+S_{1}^{-1} \sum_{m=1}^{N-1} \gamma_{m} \sum_{j \in \Omega}\left(\frac{p_{j}}{k_{m, j}} \mathrm{KI}_{1}\left(a_{m, j}\right)-\frac{p_{j}}{k_{m+1, j}} \mathrm{KI}_{1}\left(b_{m, j}\right)\right) \\
& =\gamma_{1}+\sum_{m=1}^{N-1} \gamma_{m} \sum_{j \in \Omega}\left(\frac{p_{j}}{k_{m, j}} \mathrm{KA}_{1}\left(a_{m, j}\right)-\frac{p_{j}}{k_{m+1, j}} \mathrm{KA}_{1}\left(b_{m, j}\right)\right) .
\end{aligned}
$$

For $2 \leq n \leq N-1$ we similarly obtain

$$
\begin{aligned}
0=S_{n}^{-1} \sum_{m=1}^{N-1} \gamma_{m} \mu_{n, m}= & S_{n}^{-1} \sum_{m=1}^{N-1} \gamma_{m} \sum_{j \in \Omega}\left(\frac{p_{j}}{k_{m, j}} \mathrm{KI}_{n}\left(a_{m, j}\right)-\frac{p_{j}}{k_{m+1, j}} \mathrm{KI}_{n}\left(b_{m, j}\right)\right) \\
& +S_{n}^{-1}\left(\gamma_{n} \sum_{j \in \Omega} \frac{p_{j}}{k_{n, j}}-\gamma_{n-1} \sum_{j \in \Omega} \frac{p_{j}}{k_{n, j}}\right) \\
= & S_{n}^{-1} \sum_{m=1}^{N-1} \gamma_{m} \sum_{j \in \Omega}\left(\frac{p_{j}}{k_{m, j}} \mathrm{KI}_{n}\left(a_{m, j}\right)-\frac{p_{j}}{k_{m+1, j}} \mathrm{KI}_{n}\left(b_{m, j}\right)\right) \\
& +\gamma_{n}-\gamma_{n-1} .
\end{aligned}
$$

Then summing over all $1 \leq n \leq i$ and using (16) and Lemma 3.1 gives

$$
\begin{aligned}
0 & =\sum_{n=1}^{i} S_{n}^{-1} \sum_{m=1}^{N-1} \gamma_{m} \mu_{n, m} \\
& =\gamma_{i}+\sum_{n=1}^{i} S_{n}^{-1} \sum_{m=1}^{N-1} \gamma_{m} \sum_{j \in \Omega}\left(\frac{p_{j}}{k_{m, j}} \mathrm{KI}_{n}\left(a_{m, j}\right)-\frac{p_{j}}{k_{m+1, j}} \mathrm{KI}_{n}\left(b_{m, j}\right)\right) \\
& =\gamma_{i}+\sum_{m=1}^{N-1} \gamma_{m} \sum_{j \in \Omega}\left(\frac{p_{j}}{k_{m, j}} \mathrm{KA}_{i}\left(a_{m, j}\right)-\frac{p_{j}}{k_{m+1, j}} \mathrm{KA}_{i}\left(b_{m, j}\right)\right) .
\end{aligned}
$$

This gives the relations for $\mathrm{KA}_{i}$. 
From $\sum_{m=1}^{N-1} \gamma_{m} \mu_{n, m}=0$ for all $n$ it also follows that $\sum_{m=1}^{N-1} \gamma_{m} \sum_{n=1}^{N} \mu_{n, m}=0$. From this we obtain that

$$
\sum_{m=1}^{N-1} \gamma_{m} \sum_{j \in \Omega} \frac{p_{j}}{k_{m, j}}\left(1+\sum_{n=1}^{N} \mathrm{KI}_{n}\left(a_{m, j}\right)\right)=\sum_{m=1}^{N-1} \gamma_{m} \sum_{j \in \Omega} \frac{p_{j}}{k_{m+1, j}}\left(1+\sum_{n=1}^{N} \mathrm{KI}_{n}\left(b_{m, j}\right)\right) .
$$

Then (10) from the proof of Lemma 3.2 gives that

$$
\sum_{m=1}^{N-1} \gamma_{m} \sum_{j \in \Omega} \frac{p_{j}}{k_{m, j}} \sum_{n=1}^{N} S_{n}^{-1} \mathrm{KI}_{n}\left(a_{m, j}\right)=\sum_{m=1}^{N-1} \gamma_{m} \sum_{j \in \Omega} \frac{p_{j}}{k_{m+1, j}} \sum_{n=1}^{N} S_{n}^{-1} \mathrm{KI}_{n}\left(b_{m, j}\right) .
$$

Hence, by Lemma 3.1 we get for each $i$ that

$$
\sum_{m=1}^{N-1} \gamma_{m} \sum_{j \in \Omega} \frac{p_{j}}{k_{m, j}}\left(\mathrm{KA}_{i}\left(a_{m, j}\right)+\mathrm{KB}_{i}\left(a_{m, j}\right)\right)=\sum_{m=1}^{N-1} \gamma_{m} \sum_{j \in \Omega} \frac{p_{j}}{k_{m+1, j}}\left(\mathrm{KA}_{i}\left(b_{m, j}\right)+\mathrm{KB}_{i}\left(b_{m, j}\right)\right) .
$$

This gives the orthogonal relations for $\mathrm{KB}_{i}$.

In the proofs of our main results we only use the second part of Lemma 3.6, that is, the orthogonal relations for $\mathrm{KB}_{i}$, but since we obtain the orthogonal relations for $\mathrm{KA}_{i}$ and $\mathrm{KB}_{i}$ more or less simultaneously, we have listed them both.

\section{Invariant densities for the random system $T$}

We now state our main result. For $y \in[0,1]$, define the $L^{1}(\lambda)$-function $L_{y}:[0,1] \rightarrow \mathbb{R}$ by

$$
L_{y}(x)=\sum_{t \geq 0} \sum_{\omega \in \Omega^{t}} \delta_{\omega}(y, t) \mathbf{1}_{\left[0, T_{\omega}(y)\right)}(x) .
$$

THEOREM 4.1. Let $T$ be a random piecewise linear system on the unit interval $[0,1]$ that satisfies assumptions (A1)-(A5) from $\S 2$. Let $M$ be the corresponding fundamental matrix and let $\gamma=\left(\gamma_{1}, \ldots, \gamma_{N-1}\right)^{\top}$ be a non-trivial solution of the system $M \gamma=0$. For each $1 \leq m \leq N-1$, define the function $h_{m}:[0,1] \rightarrow \mathbb{R}$ by

$$
h_{m}(x):=\sum_{\ell \in \Omega}\left[\frac{p_{\ell}}{k_{m, \ell}} L_{a_{m, \ell}}(x)-\frac{p_{\ell}}{k_{m+1, \ell}} L_{b_{m, \ell}}(x)\right] .
$$

Then a T-invariant function is given by

$$
h_{\gamma}:[0,1] \rightarrow \mathbb{R}, \quad x \mapsto \sum_{m=1}^{N-1} \gamma_{m} h_{m}(x)
$$

and $h_{\gamma} \neq 0$.

To show that $P_{T} h_{\gamma}=h_{\gamma} \lambda$-a.e. we have to determine, for each $x \in[0,1]$ and each branch $T_{i, j}$, whether or not $x$ has an inverse image in the branch $T_{i, j}$. Let

$$
x_{i, j}:=\frac{x-d_{i, j}}{k_{i, j}}
$$


be the inverse of $x$ under the map $T_{i, j}: \mathbb{R} \rightarrow \mathbb{R}$. By the definitions in (18) and (19), we have to show that

$$
\begin{aligned}
h_{\gamma}(x) & =\sum_{j \in \Omega} \sum_{i=1}^{N} \frac{p_{j}}{\left|k_{i, j}\right|} h_{\gamma}\left(x_{i, j}\right) \mathbf{1}_{I_{i}}\left(x_{i, j}\right) \\
& =\sum_{j \in \Omega} \sum_{i=1}^{N} \frac{p_{j}}{\left|k_{i, j}\right|} \mathbf{1}_{I_{i}}\left(x_{i, j}\right) \sum_{m=1}^{N-1} \gamma_{m} \sum_{\ell \in \Omega}\left(\frac{p_{\ell}}{k_{m, \ell}} L_{a_{m, \ell}}\left(x_{i, j}\right)-\frac{p_{\ell}}{k_{m+1, \ell}} L_{b_{m, \ell}}\left(x_{i, j}\right)\right) .
\end{aligned}
$$

The parts for $L_{a_{m, \ell}}$ and $L_{b_{m, \ell}}$ behave similarly. That is why we first study

$$
\sum_{j \in \Omega} \sum_{i=1}^{N} \frac{p_{j}}{\left|k_{i, j}\right|} \mathbf{1}_{I_{i}}\left(x_{i, j}\right) L_{y}\left(x_{i, j}\right)
$$

for general $y \in[0,1]$ through several lemmas. We introduce some notation to manage the long expressions. For $1 \leq i \leq N-1$, let

$$
\eta_{i}:=\sum_{j \in \Omega} \frac{p_{j}\left(\mathbf{1}_{(0, \infty)}\left(k_{i, j}\right)-a_{i, j}\right)}{k_{i, j}} \quad \text { and } \quad \phi_{i}:=\sum_{j \in \Omega} \frac{p_{j}\left(-\mathbf{1}_{(-\infty, 0)}\left(k_{i+1, j}\right)+b_{i, j}\right)}{k_{i+1, j}} .
$$

For $y \in[0,1]$ let $1 \leq n \leq N$ be the index such that $y \in I_{n}$ and set

$$
C(y):=\sum_{j \in \Omega}\left(\sum_{i=1}^{n-1} \frac{p_{j}}{\left|k_{i, j}\right|}+\frac{p_{j}}{\left|k_{n, j}\right|} \mathbf{1}_{(-\infty, 0)}\left(k_{n, j}\right)\right) .
$$

LEMmA 4.2. Let $y \in[0,1]$. Then

$$
y=\sum_{t \geq 0} \sum_{\omega \in \Omega^{t}} \delta_{\omega}(y, t) C\left(T_{\omega}(y)\right)-\sum_{i=1}^{N-1}\left(\eta_{i}+\phi_{i}\right) \mathrm{KB}_{i}(y) .
$$

Proof. Let $y \in[0,1]$ be given and recall the definition of $\theta_{\omega}(z, t)$ from (11). If $y \in I_{n}$, then

$$
\begin{aligned}
C(y) & -\sum_{i=1}^{N-1}\left(\eta_{i}+\phi_{i}\right) \mathbf{1}_{B_{i}}(y) \\
= & \sum_{j \in \Omega} \frac{p_{j}}{\left|k_{n, j}\right|} \mathbf{1}_{(-\infty, 0)}\left(k_{n, j}\right) \\
& +\sum_{j \in \Omega} \sum_{i=1}^{n-1}\left(\frac{p_{j}}{\left|k_{i, j}\right|}-\frac{p_{j}\left(\mathbf{1}_{(0, \infty)}\left(k_{i, j}\right)-a_{i, j}\right)}{k_{i, j}}-\frac{p_{j}\left(-\mathbf{1}_{(-\infty, 0)}\left(k_{i+1, j}\right)+b_{i, j}\right)}{k_{i+1, j}}\right) \\
= & \sum_{j \in \Omega}\left(-\frac{p_{j}}{k_{n, j}} b_{n-1, j}+\frac{p_{j}}{\left|k_{1, j}\right|}-\frac{p_{j}}{k_{1, j}} \mathbf{1}_{(0, \infty)}\left(k_{1, j}\right)+\frac{p_{j}}{k_{1, j}} a_{1, j}+\sum_{i=2}^{n-1} \frac{p_{j}}{k_{i, j}}\left(a_{i, j}-b_{i-1, j}\right)\right) \\
= & -\sum_{j \in \Omega} \frac{p_{j}}{k_{n, j}} d_{n, j}=\sum_{j \in \Omega} \theta_{j}(y, 1),
\end{aligned}
$$


where we have used the assumptions from (A4) in the second to last step. So for any $t \geq 0$ and $\omega \in \Omega^{t}$ we get that

$$
C\left(T_{\omega}(y)\right)-\sum_{i=1}^{N-1}\left(\eta_{i}+\phi_{i}\right) \mathbf{1}_{B_{i}}\left(T_{\omega}(y)\right)=\sum_{j \in \Omega} \theta_{\omega j}(y, t+1),
$$

where $\omega j$ denotes the concatenation of $\omega$ with $j \in \Omega$. Recall from the first line of (14) that

$$
y=\sum_{t \geq 0} \sum_{\omega \in \Omega^{t}} \delta_{\omega}(y, t) \sum_{j \in \Omega} \theta_{\omega j}(y, t+1) .
$$

Combining this with (22) and the definition of $\mathrm{KB}_{i}$ from (7) then gives the result.

For each $1 \leq i \leq N-1$, define the functions $E_{i}, F_{i}:[0,1] \rightarrow \mathbb{R}$ by

$$
\begin{aligned}
E_{i}(x) & :=\sum_{j \in \Omega} \frac{p_{j}}{k_{i, j}}\left(-\mathbf{1}_{\left[a_{i, j}, 1\right]}(x) \mathbf{1}_{(0, \infty)}\left(k_{i, j}\right)+\mathbf{1}_{\left[0, a_{i, j}\right)}(x) \mathbf{1}_{(-\infty, 0)}\left(k_{i, j}\right)\right), \\
F_{i}(x) & :=\sum_{j \in \Omega} \frac{p_{j}}{k_{i+1, j}}\left(-\mathbf{1}_{\left[0, b_{i, j}\right)}(x) \mathbf{1}_{(0, \infty)}\left(k_{i+1, j}\right)+\mathbf{1}_{\left[b_{i, j}, 1\right]}(x) \mathbf{1}_{(-\infty, 0)}\left(k_{i+1, j}\right)\right)
\end{aligned}
$$

and let $E_{N}, F_{0}:[0,1] \rightarrow \mathbb{R}$ be the zero functions. Then for each $1 \leq i \leq N$ we have that for Lebesgue almost every $x \in[0,1]$,

$$
E_{i}(x)+F_{i-1}(x)=\sum_{j \in \Omega} \frac{p_{j}}{\left|k_{i, j}\right|}\left(\mathbf{1}_{I_{i}}\left(x_{i, j}\right)-1\right),
$$

where we have used (A4) for $i=1, N$. In fact, equality holds for all but countably many points.

LEMMA 4.3. For $y \in[0,1]$ we have that for Lebesgue almost every $x \in[0,1]$,

$$
\begin{aligned}
& \sum_{j \in \Omega} \sum_{i=1}^{N} \frac{p_{j}}{\left|k_{i, j}\right|} \mathbf{1}_{I_{i}}\left(x_{i, j}\right) L_{y}\left(x_{i, j}\right) \\
& \quad=\sum_{i=1}^{N-1}\left(E_{i}(x)+\eta_{i}+F_{i}(x)+\phi_{i}\right) \mathrm{KB}_{i}(y)+y+L_{y}(x)-\mathbf{1}_{[0, y)}(x) .
\end{aligned}
$$

Proof. For $y \in[0,1]$, let $1 \leq n \leq N$ be the index such that $y \in I_{n}$. By Fubini's theorem, we get

$$
\sum_{j \in \Omega} \sum_{i=1}^{N} \frac{p_{j}}{\left|k_{i, j}\right|} \mathbf{1}_{I_{i}}\left(x_{i, j}\right) L_{y}\left(x_{i, j}\right)=\sum_{t \geq 0} \sum_{\omega \in \Omega^{t}} \delta_{\omega}(y, t) \sum_{i=1}^{N} \sum_{j \in \Omega} \frac{p_{j}}{\left|k_{i, j}\right|} \mathbf{1}_{I_{i} \cap\left[0, T_{\omega}(y)\right)}\left(x_{i, j}\right) .
$$

For Lebesgue almost every $x \in[0,1]$ and for $n \neq 1$ we have that

$$
\begin{aligned}
& \sum_{j \in \Omega} \frac{p_{j}}{\left|k_{n, j}\right|} \mathbf{1}_{(-\infty, 0)}\left(k_{n, j}\right)+\sum_{j \in \Omega} \frac{p_{j}}{k_{n, j}} \mathbf{1}_{\left[0, T_{j}(y)\right)}(x)+F_{n-1}(x) \\
& =\sum_{j \in \Omega}\left(\frac{p_{j}}{\left|k_{n, j}\right|} \mathbf{1}_{(-\infty, 0)}\left(k_{n, j}\right)\left(1-\mathbf{1}_{\left[0, T_{j}(y)\right)}(x)-\mathbf{1}_{\left[b_{n-1, j}, 1\right]}(x)\right)\right.
\end{aligned}
$$




$$
\begin{aligned}
& \left.+\frac{p_{j}}{\left|k_{n, j}\right|} \mathbf{1}_{(0, \infty)}\left(k_{n, j}\right)\left(\mathbf{1}_{\left[0, T_{j}(y)\right)}(x)-\mathbf{1}_{\left[0, b_{n-1, j}\right)}(x)\right)\right) \\
= & \sum_{j \in \Omega} \frac{p_{j}}{\left|k_{n, j}\right|} \mathbf{1}_{I_{n} \cap[0, y)}\left(x_{n, j}\right) .
\end{aligned}
$$

Using (A4), we also get that

$$
\sum_{j \in \Omega} \frac{p_{j}}{\left|k_{1, j}\right|} \mathbf{1}_{(-\infty, 0)}\left(k_{1, j}\right)+\sum_{j \in \Omega} \frac{p_{j}}{k_{1, j}} \mathbf{1}_{\left[0, T_{j}(y)\right)}(x)+F_{0}(x)=\sum_{j \in \Omega} \frac{p_{j}}{\left|k_{1, j}\right|} \mathbf{1}_{I_{1} \cap[0, y)}\left(x_{1, j}\right),
$$

so the statement from (24) holds for all $1 \leq n \leq N$. Since $y \in I_{n}$ we have for Lebesgue almost every $x \in[0,1]$ that

$$
\sum_{i=1}^{N-1}\left(E_{i}(x)+F_{i}(x)\right) \mathbf{1}_{B_{i}}(y)=\sum_{i=1}^{n-1} \sum_{j \in \Omega} \frac{p_{j}}{\left|k_{i, j}\right|}\left(\mathbf{1}_{I_{i}}\left(x_{i, j}\right)-1\right)+F_{n-1}(x) .
$$

Combining this with (24) and the definition of $C(y)$ from (21), we obtain that for each $y \in[0,1]$, there is a set of $x \in[0,1]$ of full Lebesgue measure, for which

$$
\begin{aligned}
& \sum_{j \in \Omega} \sum_{i=1}^{N} \frac{p_{j}}{\left|k_{i, j}\right|} \mathbf{1}_{I_{i} \cap[0, y)}\left(x_{i, j}\right) \\
& \quad=\sum_{j \in \Omega} \sum_{i=1}^{n-1} \frac{p_{j}}{\left|k_{i, j}\right|} \mathbf{1}_{I_{i}}\left(x_{i, j}\right)+\sum_{j \in \Omega} \frac{p_{j}}{\left|k_{n, j}\right|} \mathbf{1}_{(-\infty, 0)}\left(k_{n, j}\right)+\sum_{j \in \Omega} \frac{p_{j}}{k_{n, j}} \mathbf{1}_{\left[0, T_{j}(y)\right)}(x)+F_{n-1}(x) \\
& \quad=\sum_{i=1}^{N-1}\left(E_{i}(x)+F_{i}(x)\right) \mathbf{1}_{B_{i}}(y)+C(y)+\sum_{j \in \Omega} \tau_{j}(y, 1) \mathbf{1}_{\left[0, T_{j}(y)\right)}(x) .
\end{aligned}
$$

Hence, by (23) we also have that for Lebesgue almost every $x \in[0,1]$,

$$
\begin{aligned}
\sum_{j \in \Omega} & \sum_{i=1}^{N} \frac{p_{j}}{\left|k_{i, j}\right|} \mathbf{1}_{I_{i}}\left(x_{i, j}\right) L_{y}\left(x_{i, j}\right) \\
= & \sum_{i=1}^{N-1}\left(E_{i}(x)+F_{i}(x)\right) \sum_{t \geq 0} \sum_{\omega \in \Omega^{t}} \delta_{\omega}(y, t) \mathbf{1}_{B_{i}}\left(T_{\omega}(y)\right) \\
& +\sum_{t \geq 0} \sum_{\omega \in \Omega^{t}} \delta_{\omega}(y, t) C\left(T_{\omega}(y)\right)+\sum_{t \geq 1} \sum_{\omega \in \Omega^{t}} \delta_{\omega}(y, t) \mathbf{1}_{\left[0, T_{\omega}(y)\right)}(x) .
\end{aligned}
$$

The statement now follows from the definition of $\mathrm{KB}_{i}$ from (7) and Lemma 4.2.

Proof of Theorem 4.1. First note that for all $1 \leq i \leq N-1$ and all $x \in[0,1]$,

$$
E_{i}(x)+\eta_{i}=\sum_{j \in \Omega} \frac{p_{j}}{k_{i, j}}\left(\mathbf{1}_{\left[0, a_{i, j}\right)}(x)-a_{i, j}\right)
$$

and

$$
F_{i}(x)+\phi_{i}=\sum_{j \in \Omega} \frac{p_{j}}{k_{i+1, j}}\left(-\mathbf{1}_{\left[0, b_{i, j}\right.}(x)+b_{i, j}\right) .
$$


Together they give that

$$
\begin{aligned}
& \sum_{\ell \in \Omega}\left(\frac{p_{\ell}}{k_{m, \ell}}\left(-\mathbf{1}_{\left[0, a_{m, \ell}\right)}(x)+a_{m, \ell}\right)-\frac{p_{\ell}}{k_{m+1}, \ell}\left(-\mathbf{1}_{\left[0, b_{m, \ell}\right)}(x)+b_{m, \ell}\right)\right) \\
& \quad=-\left(E_{m}(x)+\eta_{m}+F_{m}(x)+\phi_{m}\right) .
\end{aligned}
$$

Using this together with Lemma 4.3 and Fubini's theorem, we get by (20) that for Lebesgue almost every $x \in[0,1]$,

$$
\begin{aligned}
P_{T} & h_{\gamma}(x) \\
= & \sum_{m=1}^{N-1} \gamma_{m} \sum_{i=1}^{N-1}\left(E_{i}(x)+\eta_{i}+F_{i}(x)+\phi_{i}\right) \sum_{\ell \in \Omega}\left(\frac{p_{\ell}}{k_{m, \ell}} \operatorname{KB}_{i}\left(a_{m, \ell}\right)-\frac{p_{\ell}}{k_{m+1, \ell}} \operatorname{KB}_{i}\left(b_{m, \ell}\right)\right) \\
& -\sum_{m=1}^{N-1} \gamma_{m}\left(E_{m}(x)+\eta_{m}+F_{m}(x)+\phi_{m}\right)+h_{\gamma}(x) .
\end{aligned}
$$

From the second part of Lemma 3.6 we can deduce by multiplying by $E_{i}(x)+\eta_{i}+$ $F_{i}(x)+\phi_{i}$ and summing over all $i$ that

$$
\begin{aligned}
& \sum_{i=1}^{N-1}\left(E_{i}(x)+\eta_{i}+F_{i}(x)+\phi_{i}\right) \gamma_{i} \\
& \quad=\sum_{i=1}^{N-1}\left(E_{i}(x)+\eta_{i}+F_{i}(x)+\phi_{i}\right) \sum_{m=1}^{N-1} \gamma_{m} \sum_{j \in \Omega}\left(\frac{p_{j}}{k_{m, j}} \mathrm{~KB}_{i}\left(a_{m, j}\right)-\frac{p_{j}}{k_{m+1, j}} \mathrm{~KB}_{i}\left(b_{m, j}\right)\right) .
\end{aligned}
$$

Hence, we have obtained that $h_{\gamma}$ is a $T$-invariant function in $L^{1}(\lambda)$.

It remains to show that $h_{\gamma} \neq 0$. Recall from $\S 2$ that any $T$-invariant $L^{1}(\lambda)$-function is of bounded variation. So at any point $y \in[0,1]$ the $\operatorname{limits}_{\lim _{x \uparrow y}} h_{\gamma}(x)$ and $\lim _{x \downarrow y} h_{\gamma}(x)$ exist. Consider $1 \leq \ell \leq N-1$ and assume $z_{\ell} \in I_{\ell}$. Then for all $y \in[0,1]$, by (6) and (7), we obtain, by the dominated convergence theorem,

$$
\lim _{x \downarrow z \ell} L_{y}(x)=\sum_{t \geq 0} \sum_{\omega \in \Omega^{t}} \delta_{\omega}(y, t) \lim _{x \downarrow z \ell} \mathbf{1}_{\left[0, T_{\omega}(y)\right)}(x)=\sum_{t \geq 0} \sum_{\omega \in \Omega^{t}} \delta_{\omega}(y, t) \mathbf{1}_{B_{\ell}}\left(T_{\omega}(y)\right)=\operatorname{KB}_{\ell}(y) .
$$

From this, Lemma 3.6 and the dominated convergence theorem again we then get

$$
\begin{aligned}
\lim _{x \downarrow z \ell} h_{\gamma}(x) & =\sum_{m=1}^{N-1} \gamma_{m} \sum_{j \in \Omega} \lim _{x \downarrow z \ell}\left[\frac{p_{j}}{k_{m, j}} L_{a_{m, j}}(x)-\frac{p_{j}}{k_{m+1, j}} L_{b_{m, j}}(x)\right] \\
& =\sum_{m=1}^{N-1} \gamma_{m} \sum_{j \in \Omega}\left[\frac{p_{j}}{k_{m, j}} \operatorname{KB}_{\ell}\left(a_{m, j}\right)-\frac{p_{j}}{k_{m+1, j}} \operatorname{KB}_{\ell}\left(b_{m, j}\right)\right] \\
& =\gamma_{\ell} .
\end{aligned}
$$

If, on the other hand, $z_{\ell} \in I_{\ell+1}$, then we obtain similarly that $\lim _{x \uparrow z_{\ell}} L_{y}(x)=\mathrm{KB}_{\ell}(y)$ and thus that $\lim _{x \uparrow z_{\ell}} h_{\gamma}(x)=\gamma_{\ell}$. Hence, $h_{\gamma}=0$ implies $\gamma=0$. This proves the theorem. 
Remark 4.4. Theorem 4.1 assigns to each solution $\gamma \neq 0$ of $M \gamma=0$ a $T$-invariant $L^{1}(\lambda)$-function $h_{\gamma} \neq 0$. From $h_{\gamma}$ we can get invariant densities for $T$ as follows. If $h_{\gamma}$ is positive or negative, then we can scale $h_{\gamma}$ to an invariant density function. If not, then we can write $h_{\gamma}=h^{+}-h^{-}$for two positive functions $h^{+}:[0,1] \rightarrow[0, \infty)$ and $h^{-}:[0,1] \rightarrow[0, \infty)$ and by the linearity and positivity of $P_{T}$ it follows that

$$
h^{+}-h^{-}=h_{\gamma}=P_{T} h_{\gamma}=P_{T} h^{+}-P_{T} h^{-} .
$$

Hence, $h^{+}$and $h^{-}$can both be normalized to obtain invariant densities for $T$.

Remark 4.5. In order to compute $h_{\gamma}$, one needs to compute the fundamental matrix $M$ and a vector $\gamma$ first. Lemma 3.4 implies that when $N$ is small, the computation of $\gamma$ is straightforward. Indeed, for $N=2, M$ is the null vector, and we can take $\gamma=1$. This is illustrated by the example of the random tent maps from $\S 6.1$. For $N=3$, it is enough to compute only one row of $M$ and take $\gamma=\left(\begin{array}{c}-\mu_{i, 2} \\ \mu_{i, 1}\end{array}\right)^{\top}$. We see an illustration of this fact in $\S 6.3$ and $\S 6.4$ on random $\beta$-transformations. For larger $N$, the computation of $M$ can still be simplified by using the relations from Lemma 3.2.

To end this section we give a small example to show that condition (A5) is not necessary for Theorem 4.1 to hold. Consider the random system with $\Omega=\{0,1\}, T_{0}(x)=$ $2 x(\bmod 1)$ the doubling map, $T_{1}(x)=1-T_{0}(x)$ and $p_{0}=p_{1}=\frac{1}{2}$. Then $N=2$ and for both $n=1,2$ we have $S_{n}=\frac{1}{2} \cdot \frac{1}{2}-\frac{1}{2} \cdot \frac{1}{2}=0$. Hence $M=\left(\begin{array}{c}0 \\ 0\end{array}\right)^{\top}$ and any $\gamma=\gamma_{1} \in$ $\mathbb{R} \backslash\{0\}$ is a non-trivial solution to $M \gamma=0$. Since all critical points of $T_{0}$ and $T_{1}$ are mapped to 0 or 1 , the function $h_{1}$ from (18) will be of the form $c \cdot \mathbf{1}_{[0,1)}$ for some $c \neq 0$ and the function $h_{\gamma}=\gamma / c \cdot \mathbf{1}_{[0,1)}$ is indeed invariant for $T$.

\section{All possible absolutely continuous invariant measures}

The aim of this section is twofold. Firstly, we prove that the way $T$ is defined on the partition points $z_{\ell}$ does not influence the final result. In other words, the set of invariant functions we obtain from Theorem 4.1 if $z_{\ell} \in I_{\ell}$ is equal to the set of invariant functions we obtain if we choose $z_{\ell} \in I_{\ell+1}$. This is the content of Proposition 5.1. The amount of work it takes to compute the matrix $M$ and the invariant functions $h_{\gamma}$ depends on whether $z_{\ell} \in I_{\ell}$ or $z_{\ell} \in I_{\ell+1}$. Proposition 5.1 tells us that we are free to choose the most convenient option. We shall see several examples below. Next we will use Proposition 5.1 to prove that, under the additional assumption that all maps $T_{j}$ are expanding, Theorem 4.1 actually produces all absolutely continuous invariant measures of $T$. We do this by proving in Theorem 5.3 that the map $\gamma \mapsto h_{\gamma}$ is a bijection between the null space of $M$ and the subspace of $L^{1}(\lambda)$ of all $T$-invariant functions.

PROPOSITION 5.1. Let $T$ be a random system with partition $\left\{I_{i}\right\}_{1 \leq i \leq N}$ and corresponding partition points $z_{0}, \ldots, z_{N}$. Let $\left\{\hat{I}_{i}\right\}_{1 \leq i \leq N}$ be another partition of $[0,1]$ given by $z_{0}, \ldots, z_{N}$ and differing from $\left\{I_{i}\right\}_{1 \leq i \leq N}$ only in one or more of the points $z_{1}, \ldots, z_{N-1}$. Let $\hat{T}$ be the corresponding random system, that is, $\hat{T}(x)=T(x)$ for all $x \neq z_{i}, 1 \leq i \leq$ $N-1$. Let $\hat{M}$ be the fundamental matrix of $\hat{T}$. There is a one-to-one correspondence between the solutions $\gamma$ of $M \gamma=0$ and the solutions $\hat{\gamma}$ of $\hat{M} \hat{\gamma}=0$. Moreover, the functions $h_{\gamma}$ and $\hat{h}_{\hat{\gamma}}$ coincide. 
Proof. First assume that there is only one point $z_{\ell}$ on which $\left\{I_{i}\right\}_{1 \leq i \leq N}$ and $\left\{\hat{I}_{i}\right\}_{1 \leq i \leq N}$ differ. We show that any column of $\hat{M}$ is a linear combination of columns of $M$. More precisely, we show that the $i$ th column of $\hat{M}$ is a linear combination of the $i$ th and the $\ell$ th columns of $M$. Assume without loss of generality that $z_{\ell} \in I_{\ell}$ and therefore $z_{\ell} \in \hat{I}_{\ell+1}$. This implies that $T_{j}\left(z_{\ell}\right)=a_{\ell, j}$, whereas $\hat{T}_{j}\left(z_{\ell}\right)=b_{\ell, j}$. This difference is reflected in the values of the quantities $\mathrm{KI}_{n}\left(a_{i, s}\right)$ and $\mathrm{KI}_{n}\left(b_{i, s}\right)$ appearing in the matrix $M$ if $a_{i, s}$ or $b_{i, s}$ enters $z_{\ell}$ under some iteration of $T$. We will describe these changes, but first we define some quantities.

For any $y \in\left\{a_{i, j}, b_{i, j}: 1 \leq i \leq N-1, j \in \Omega\right\}$ let $\Omega_{y} \subseteq \Omega^{*}$ be the collection of paths that lead $y$ to $z_{\ell}$, that is, $\omega \in \Omega_{y}$ if and only if there is a $0 \leq t<|\omega|$, such that $T_{\omega_{1}^{t}}(y)=z_{\ell}$. Let

$$
\Omega_{y}^{t}:=\left\{\omega \in \Omega^{*} \mid \exists \eta \in \Omega_{y}: \omega=\eta_{1}^{t}, T_{\omega}(y)=z_{\ell} \text { and } T_{\omega_{1}^{s}}(y) \neq z_{\ell} \text { for } s<t\right\} .
$$

Then $\Omega_{y}^{t}$ is the collection of words of length $t$ that lead $y$ to $z_{\ell}$ via a path that does not lead $y$ to $z_{\ell}$ before time $t$. We are interested in the difference between the quantities $\operatorname{KI}_{n}(y)$ and $\mathrm{K}_{n}(y)$ and we let $C_{n}^{y}$ denote the part that they have in common, that is, set

$$
C_{n}^{y}:=\sum_{t \geq 1} \sum_{\omega \in \Omega_{y}^{t} \cup \Omega^{t} \backslash \Omega_{y}} \delta_{\omega}(y, t) \mathbf{1}_{I_{n}}\left(T_{\omega_{1}^{t-1}}(y)\right) .
$$

Then for $n \neq \ell$, we get

$$
\begin{aligned}
\mathrm{KI}_{n}(y) & =C_{n}^{y}+\sum_{t \geq 0} \sum_{\omega \in \Omega_{y}^{t}} \sum_{u \geq 1} \sum_{\eta \in \Omega^{u}} \delta_{\omega}(y, t) \delta_{\eta}\left(z_{\ell}, u\right) \mathbf{1}_{I_{n}}\left(T_{\eta_{1}^{u-1}}\left(z_{\ell}\right)\right) \\
& =C_{n}^{y}+\sum_{t \geq 0} \sum_{\omega \in \Omega_{y}^{t}} \sum_{u \geq 1} \sum_{\eta \in \Omega^{u}} \sum_{j \in \Omega} \delta_{\omega}(y, t) \frac{p_{j}}{k_{\ell, j}} \delta_{\eta}\left(a_{\ell, j}, u\right) \mathbf{1}_{I_{n}}\left(T_{\eta_{1}^{u-1}}\left(a_{\ell, j}\right)\right) \\
& =C_{n}^{y}+\sum_{t \geq 0} \sum_{\omega \in \Omega_{y}^{t}} \delta_{\omega}(y, t) \sum_{j \in \Omega} \frac{p_{j}}{k_{\ell, j}} \mathrm{KI}_{n}\left(a_{\ell, j}\right),
\end{aligned}
$$

and similarly, for $n=\ell$ we obtain

$$
\mathrm{KI}_{\ell}(y)=C_{\ell}^{y}+\sum_{t \geq 0} \sum_{\omega \in \Omega_{y}^{t}} \delta_{\omega}(y, t) \sum_{j \in \Omega} \frac{p_{j}}{k_{\ell, j}}\left(1+\mathrm{KI}_{\ell}\left(a_{\ell, j}\right)\right) .
$$

If we set $Q(y)=\sum_{t \geq 0} \sum_{\omega \in \Omega_{y}^{t}} \delta_{\omega}(y, t)$ as the constant that keeps track of all the paths that lead $y$ to $z_{\ell}$ for the first time, then we can write

$$
\begin{aligned}
& \mathrm{KI}_{n}(y)=C_{n}^{y}+Q(y) \sum_{j \in \Omega} \frac{p_{j}}{k_{\ell, j}} \mathrm{KI}_{n}\left(a_{\ell, j}\right) \text { for } n \neq \ell, \\
& \mathrm{KI}_{\ell}(y)=C_{\ell}^{y}+Q(y) \sum_{j \in \Omega} \frac{p_{j}}{k_{\ell, j}}\left(1+\mathrm{KI}_{\ell}\left(a_{\ell, j}\right)\right) .
\end{aligned}
$$


On the other hand, for $\mathrm{KI}_{n}(y)$ we get

$$
\begin{aligned}
\mathrm{K}_{n}(y) & =C_{n}^{y}+Q(y) \sum_{j \in \Omega} \frac{p_{j}}{k_{\ell+1, j}} \mathrm{~K}_{n}\left(b_{\ell, j}\right) \quad \text { for } n \neq \ell+1, \\
\mathrm{~K}_{\ell+1}(y) & =C_{\ell+1}^{y}+Q(y) \sum_{j \in \Omega} \frac{p_{j}}{k_{\ell+1, j}}\left(1+\mathrm{K}_{\ell+1}\left(b_{\ell, j}\right)\right) .
\end{aligned}
$$

If $b_{\ell, j}$ does not return to $z_{\ell}$, then $\mathrm{KI}_{n}\left(b_{\ell, j}\right)=\mathrm{K}_{n}\left(b_{\ell, j}\right)$. Set

$$
B:=\left\{j \in \Omega: \Omega_{b_{\ell, j}} \neq \emptyset\right\} .
$$

Then

$$
\begin{aligned}
\mathrm{K}_{n}(y)= & C_{n}^{y}+Q(y) \sum_{j \notin B} \frac{p_{j}}{k_{\ell+1, j}} \mathrm{KI}_{n}\left(b_{\ell, j}\right) \\
& +Q(y) \sum_{j \in B} \frac{p_{j}}{k_{\ell+1, j}} \mathrm{~K}_{n}\left(b_{\ell, j}\right) \quad \text { for } n \neq \ell+1, \\
\mathrm{~K}_{\ell+1}(y)= & C_{\ell+1}^{y}+Q(y) \sum_{j \notin B} \frac{p_{j}}{k_{\ell+1, j}}\left(1+\mathrm{KI}_{\ell+1}\left(b_{\ell, j}\right)\right) \\
& +Q(y) \sum_{j \in B} \frac{p_{j}}{k_{\ell+1, j}}\left(1+\mathrm{K}_{\ell+1}\left(b_{\ell, j}\right)\right) .
\end{aligned}
$$

To determine the difference between $\mathrm{KI}_{n}(y)$ and $\mathrm{K}_{n}(y)$, we would like an expression for $\mathrm{K}_{n}\left(b_{\ell, j}\right)$ in terms of $\mathrm{KI}_{n}\left(b_{\ell, j}\right)$ for $j \in B$. Fix $n \neq \ell+1$ for a moment and set, for each $j \in B$,

$$
A_{j}=C_{n}^{b_{\ell, j}}+Q\left(b_{\ell, j}\right) \sum_{i \notin B} \frac{p_{i}}{k_{\ell+1, i}} \mathrm{KI}_{n}\left(b_{\ell, i}\right)
$$

Then we can find expressions for $\mathrm{K}_{n}\left(b_{\ell, j}\right)$ in terms of the values $\mathrm{KI}_{n}\left(b_{\ell, i}\right)$ by solving the following system of linear equations:

$$
\mathrm{K}_{n}\left(b_{\ell, j}\right)=A_{j}+Q\left(b_{\ell, j}\right) \sum_{i \in B} \frac{p_{i}}{k_{\ell+1, i}} \mathrm{~K}_{n}\left(b_{\ell, i}\right), \quad j \in B .
$$

A solution is easily computed through Cramer's method, which gives, for $j \in B$,

$$
\mathrm{K}_{n}\left(b_{\ell, j}\right)=\frac{A_{j}\left(1-\sum_{u \in B \backslash\{j\}} Q\left(b_{\ell, u}\right)\left(p_{u} / k_{\ell+1, u}\right)\right)+Q\left(b_{\ell, j}\right) \sum_{u \in B \backslash\{j\}}\left(p_{u} / k_{\ell+1, u}\right) A_{u}}{1-\sum_{i \in B} Q\left(b_{\ell, i}\right)\left(p_{i} / k_{\ell+1, i}\right)} .
$$

Set

$$
B_{\ell}:=1-\sum_{j \in \Omega} Q\left(b_{\ell, j}\right) \frac{p_{j}}{k_{\ell+1, j}} .
$$

Below we will use $B_{\ell}^{-1}$. If $\left|Q\left(b_{\ell, j}\right)\right| \leq 1$, then

$$
\left|\sum_{j \in \Omega} Q\left(b_{\ell, j}\right) \frac{p_{j}}{k_{\ell+1, j}}\right| \leq \sum_{j \in \Omega}\left|Q\left(b_{\ell, j}\right)\right| \frac{p_{j}}{\left|k_{\ell+1, j}\right|} \leq \sum_{j \in \Omega} \frac{p_{j}}{\left|k_{\ell+1, j}\right|} \leq \rho<1,
$$


so in this case $B_{\ell} \neq 0$ and $B_{\ell}^{-1}$ is well defined. We now show that $\left|Q\left(b_{\ell, j}\right)\right| \leq 1$. If $b_{\ell, j}=z_{\ell}$, then $\Omega_{b_{\ell, j}}^{t}=\emptyset$ for any $t \geq 1$, and so $Q\left(b_{\ell, j}\right)=1$. If $b_{\ell, j} \neq z_{\ell}$, then $Q\left(b_{\ell, j}\right)=$ $\sum_{t \geq 1} \sum_{\omega \in \Omega_{b_{\ell, j}}^{t}} \delta_{\omega}\left(b_{\ell, j}, t\right)$. By the expanding on average property (A2), for any $y \in I$, any $t \geq 0$ and any $\omega \in \Omega^{\mathbb{N}}$,

$$
\left|\delta_{\omega}(y, t)\right|>\sum_{j \in \Omega}\left|\delta_{\omega}(y, t) \tau_{j}\left(T_{\omega_{1}^{t}}(y), 1\right)\right|=\sum_{j \in \Omega}\left|\delta_{\omega j}(y, t+1)\right| .
$$

Note that by the definition of $Q\left(b_{\ell, j}\right)$ the union

$$
\bigcup_{t \geq 1} \bigcup_{\omega \in \Omega_{b_{\ell, j}^{t}}^{t}}[\omega] \subseteq \Omega^{\mathbb{N}}
$$

is a disjoint union of cylinder sets. Hence, by repeated application of (29) we obtain for each $n \geq 1$ that

$$
\begin{aligned}
1=\left|\delta_{\epsilon}\left(b_{\ell, j}, 0\right)\right| & >\sum_{i_{1} \in \Omega}\left|\delta_{i_{1}}\left(b_{\ell, j}, 1\right)\right|=\sum_{i_{1} \in \Omega_{b_{\ell, j}}}\left|\delta_{i_{1}}\left(b_{\ell, j}, 1\right)\right|+\sum_{i_{1} \in \Omega_{b_{\ell, j}^{c}}^{c}}\left|\delta_{i_{1}}\left(b_{\ell, j}, 1\right)\right| \\
& >\sum_{i_{1} \in \Omega_{b_{\ell, j}}}\left|\delta_{i_{1}}\left(b_{\ell, j}, 1\right)\right|+\sum_{i_{1} \in \Omega_{b_{\ell, j}}^{c}} \sum_{i_{2} \in \Omega}\left|\delta_{i_{1} i_{2}}\left(b_{\ell, j}, 2\right)\right| \\
& =\sum_{t=1}^{2} \sum_{\omega \in \Omega_{b_{\ell, j}}^{t}}\left|\delta_{\omega}\left(b_{\ell, j}, t\right)\right|+\sum_{\omega \in\left(\Omega_{b_{\ell, j}} \cup \Omega_{b_{\ell, j}}^{2}\right)^{c}}\left|\delta_{\omega}\left(b_{\ell, j}, 2\right)\right| \\
& >\cdots>\sum_{t=1}^{n} \sum_{\omega \in \Omega_{b_{\ell, j}}^{t}}\left|\delta_{\omega}\left(b_{\ell, j}, t\right)\right|+\sum_{\omega \in\left(\cup_{t=1}^{n} \Omega_{b_{\ell, j}}^{t}\right)^{c}}\left|\delta_{\omega}\left(b_{\ell, j}, n\right)\right| .
\end{aligned}
$$

Since this holds for each $n$, we get $\left|Q\left(b_{\ell, j}\right)\right| \leq 1$ and $B_{\ell} \neq 0$.

For $i \notin B$ we have that $\mathrm{KI}_{n}\left(b_{\ell, i}\right)=C_{n}^{b_{\ell, i}}$. Then, by the definition of $B_{\ell}$, we get

$$
\begin{aligned}
\sum_{j \in B} \frac{p_{j}}{k_{\ell+1, j}} \mathrm{~K}_{n}\left(b_{\ell, j}\right) & =B_{\ell}^{-1} \sum_{j \in B} \frac{p_{j}}{k_{\ell+1, j}} A_{j} \\
& =B_{\ell}^{-1} \sum_{j \in B} \frac{p_{j}}{k_{\ell+1, j}}\left(C_{n}^{b_{\ell, j}}+Q\left(b_{\ell, j}\right) \sum_{i \notin B} \frac{p_{i}}{k_{\ell+1, i}} C_{n}^{b_{\ell, i}}\right) \\
& =B_{\ell}^{-1} \sum_{j \in B} \frac{p_{j}}{k_{\ell+1, j}} C_{n}^{b_{\ell, j}}+B_{\ell}^{-1}\left(1-B_{\ell}\right) \sum_{i \notin B} \frac{p_{i}}{k_{\ell+1, i}} C_{n}^{b_{\ell, i}} \\
& =B_{\ell}^{-1} \sum_{j \in \Omega} \frac{p_{j}}{k_{\ell+1, j}} C_{n}^{b_{\ell, j}}-\sum_{i \notin B} \frac{p_{i}}{k_{\ell+1, i}} C_{n}^{b_{\ell, i}} .
\end{aligned}
$$

We obtain similar expressions for $n=\ell+1$. For each $1 \leq i \leq N-1$, let

$$
Q_{i}:=\sum_{j \in \Omega}\left(\frac{p_{j}}{k_{i, j}} Q\left(a_{i, j}\right)-\frac{p_{j}}{k_{i+1, j}} Q\left(b_{i, j}\right)\right) .
$$


We show that for each $1 \leq n \leq N$ and $1 \leq i \leq N-1$ we have

$$
\hat{\mu}_{n, i}=\mu_{n, i}-Q_{i} B_{\ell}^{-1} \mu_{n, \ell},
$$

that is, the $i$ th column of $\hat{M}$ is a linear combination of the $i$ th and $\ell$ th columns of $M$. We give the proof only for $n \notin\{\ell, \ell+1, i, i+1\}$, since the other cases are very similar. To prove this, we first rewrite $\mu_{n, i}-Q_{i} B_{\ell}^{-1} \mu_{n, \ell}$. Therefore, note that

$$
\begin{aligned}
& \sum_{j \in \Omega} \frac{p_{j}}{k_{\ell, j}} \mathrm{KI}_{n}\left(a_{\ell, j}\right)-B_{\ell}^{-1}\left(\sum_{j \in \Omega} \frac{p_{j}}{k_{\ell, j}} \mathrm{KI}_{n}\left(a_{\ell, j}\right)-\sum_{j \in B} \frac{p_{j}}{k_{\ell+1, j}} Q\left(b_{\ell, j}\right) \sum_{i \in \Omega} \frac{p_{i}}{k_{\ell, i}} \mathrm{KI}_{n}\left(a_{\ell, i}\right)\right) \\
& \quad=\sum_{j \in \Omega} \frac{p_{j}}{k_{\ell, j}} \mathrm{KI}_{n}\left(a_{\ell, j}\right)\left(1-B_{\ell}^{-1} B_{\ell}\right)=0 .
\end{aligned}
$$

Then we obtain from the definition of $M,(26)$ and the above equation that

$$
\begin{aligned}
\mu_{n, i}-Q_{i} B_{\ell}^{-1} \mu_{n, \ell}= & \sum_{j \in \Omega}\left(\frac{p_{j}}{k_{i, j}} C_{n}^{a_{i, j}}-\frac{p_{j}}{k_{i+1, j}} C_{n}^{b_{i, j}}\right)+Q_{i} \sum_{j \in \Omega} \frac{p_{j}}{k_{\ell, j}} \mathrm{KI}_{n}\left(a_{\ell, j}\right) \\
& -Q_{i} B_{\ell}^{-1} \sum_{j \in \Omega} \frac{p_{j}}{k_{\ell, j}} \mathrm{KI}_{n}\left(a_{\ell, j}\right)+Q_{i} B_{\ell}^{-1} \sum_{j \notin B} \frac{p_{j}}{k_{\ell+1, j}} \mathrm{KI}_{n}\left(b_{\ell, j}\right) \\
& +Q_{i} B_{\ell}^{-1} \sum_{j \in B} \frac{p_{j}}{k_{\ell+1, j}}\left(C_{n}^{b_{\ell, j}}+Q\left(b_{\ell, j}\right) \sum_{u \in \Omega} \frac{p_{u}}{k_{\ell, u}} \mathrm{KI}_{n}\left(a_{\ell, u}\right)\right) \\
= & \sum_{j \in \Omega}\left(\frac{p_{j}}{k_{i, j}} C_{n}^{a_{i, j}}-\frac{p_{j}}{k_{i+1, j}} C_{n}^{b_{i, s}}\right)+Q_{i} B_{\ell}^{-1} \sum_{j \in \Omega} \frac{p_{j}}{k_{\ell+1, j}} C_{n}^{b_{\ell, j}} .
\end{aligned}
$$

For $\hat{\mu}_{n, i}$ we get by combining (27) and (31) that

$$
\begin{aligned}
\hat{\mu}_{n, i}= & \sum_{j \in \Omega}\left(\frac{p_{j}}{k_{i, j}} C_{n}^{a_{i, j}}+\frac{p_{j}}{k_{i+1, j}} C_{n}^{b_{i, j}}\right)+Q_{i} \sum_{j \notin B} \frac{p_{j}}{k_{\ell+1, j}} \mathrm{KI}_{n}\left(b_{\ell, j}\right) \\
& +Q_{i} B_{\ell}^{-1} \sum_{j \in \Omega} \frac{p_{j}}{k_{\ell+1, j}} C_{n}^{b_{\ell, j}}-Q_{i} \sum_{j \notin B} \frac{p_{j}}{k_{\ell+1, j}} C_{n}^{b_{\ell, j}}=\mu_{n, i}-Q_{i} B_{\ell}^{-1} \mu_{n, \ell} .
\end{aligned}
$$

One can now easily check that if $\gamma=\left(\gamma_{1}, \ldots, \gamma_{N-1}\right)^{\top}$ is a solution of $M \gamma=0$, then the vector $\hat{\gamma}=\left(\hat{\gamma}_{1}, \ldots, \hat{\gamma}_{N-1}\right)^{\top}$ given by

$$
\hat{\gamma}_{\ell}=\gamma_{\ell}+\sum_{i=1}^{N-1} \frac{Q_{i}}{B_{\ell}-Q_{\ell}} \gamma_{i}
$$

and $\hat{\gamma}_{i}=\gamma_{i}$ if $i \neq \ell$, satisfies $\hat{M} \hat{\gamma}=0$. The fact that $B_{\ell}-Q_{\ell} \neq 0$ follows in the same way as that $B_{\ell} \neq 0$. Hence, there is a one-to-one relation between the solutions $\gamma$ of $M \gamma=0$ and $\hat{\gamma}$ of $\hat{M} \hat{\gamma}=0$.

It remains to prove that the functions $h_{\gamma}$ and $\hat{h}_{\hat{\gamma}}$ coincide. For that we need to consider the functions $L_{y}$. As we did for $\mathrm{KI}_{n}$, let $L^{y}$ denote the parts that $L_{y}$ and $\hat{L}_{y}$ have in common, that is, set

$$
L^{y}=\sum_{t \geq 0} \sum_{\omega \in \Omega_{y}^{t} \cup \Omega^{t} \backslash \Omega_{y}} \delta_{\omega}(y, t) \mathbf{1}_{\left[0, T_{\omega}(y)\right)} .
$$


Set $A:=\left\{j \in \Omega: \Omega_{a_{\ell, j}} \neq \emptyset\right\}$. Then

$$
\begin{aligned}
L_{y} & =L^{y}+Q(y) \sum_{t \geq 1} \sum_{\omega \in \Omega^{t}} \delta_{\omega}\left(z_{\ell}, t\right) \mathbf{1}_{\left[0, \hat{T}_{\omega}\left(z_{\ell}\right)\right)} \\
& =L^{y}+Q(y)\left(\sum_{j \in \Omega} \mathbf{1}_{\left[0, a_{\ell, j}\right)}+\sum_{t \geq 1} \sum_{\omega \in \Omega^{t}} \frac{p_{j}}{k_{\ell, j}} \delta_{\omega}\left(b_{\ell, j}, u\right) \mathbf{1}_{\left[0, \hat{T}_{\omega}\left(a_{\ell, j}\right)\right)}\right) \\
& =L^{y}+Q(y) \sum_{j \notin A} \frac{p_{j}}{k_{\ell, j}} L_{a_{\ell, j}}+Q(y) \sum_{j \in A} \frac{p_{j}}{k_{\ell, j}} L_{a_{\ell, j}} .
\end{aligned}
$$

By Cramer's rule we obtain for each $j \in A$, that (compare (31))

$$
\sum_{j \in A} \frac{p_{j}}{k_{\ell, j}} L_{a_{\ell, j}}=\left(B_{\ell}-Q_{\ell}\right)^{-1} \sum_{j \in \Omega} \frac{p_{j}}{k_{\ell, j}} L^{a_{\ell, j}}-\sum_{j \notin A} \frac{p_{j}}{k_{\ell, j}} L_{a_{\ell, j}} .
$$

Similarly, we obtain that

$$
\hat{L}_{y}=L^{y}+Q(y) \sum_{j \notin B} \frac{p_{j}}{k_{\ell+1, j}} L_{b_{\ell, j}}+Q(y) \sum_{j \in B} \frac{p_{j}}{k_{\ell+1, j}} \hat{L}_{b_{\ell, j}}
$$

and

$$
\sum_{j \in B} \frac{p_{j}}{k_{\ell+1, j}} \hat{L}_{b_{\ell, j}}=B_{\ell}^{-1} \sum_{j \in \Omega} \frac{p_{j}}{k_{\ell+1, j}} L^{b_{\ell, j}}-\sum_{j \notin B} \frac{p_{j}}{k_{\ell+1, j}} L^{b_{\ell, j}} .
$$

To prove that $h_{\gamma}=\hat{h}_{\hat{\gamma}}$, note that, on the one hand,

$$
h_{\gamma}=\sum_{m=1}^{N-1} \gamma_{m} \sum_{j \in \Omega}\left(\frac{p_{j}}{k_{m, j}} L^{a_{m, j}}-\frac{p_{j}}{k_{m+1, j}} L^{b_{m, j}}\right)+\sum_{m=1}^{N-1} \gamma_{m} Q_{m} \sum_{j \in \Omega} \frac{p_{j}}{k_{\ell, j}} L_{a_{\ell, j}} .
$$

On the other hand, using equations (32), (34) and (35), we obtain for $\hat{h}_{\hat{\gamma}}$ that

$$
\begin{aligned}
\hat{h}_{\hat{\gamma}}= & \sum_{m=1}^{N-1} \gamma_{m} \sum_{s \in \Omega}\left(\frac{p_{s}}{k_{m, s}} L^{a_{m, s}}-\frac{p_{s}}{k_{m+1, s}} L^{b_{m, s}}\right)+\sum_{m=1}^{N-1} \gamma_{m} Q_{m}\left(1+\frac{Q_{\ell}}{B_{\ell}-Q_{\ell}}\right) \sum_{s \in \Omega} \frac{p_{s}}{k_{\ell+1, s}} \hat{L}_{b_{\ell, s}} \\
& +\sum_{m=1}^{N-1} \gamma_{m} \frac{Q_{m}}{B_{\ell}-Q_{\ell}} \sum_{s \in \Omega}\left(\frac{p_{s}}{k_{\ell, s}} L^{a_{\ell, s}}-\frac{p_{s}}{k_{\ell+1, s}} L^{b_{\ell, s}}\right) \\
= & \sum_{m=1}^{N-1} \gamma_{m} \sum_{s \in \Omega}\left(\frac{p_{s}}{k_{m, s}} L^{a_{m, s}}-\frac{p_{s}}{k_{m+1, s}} L^{b_{m, s}}\right)+\sum_{m=1}^{N-1} \gamma_{m} Q_{m} \frac{B_{\ell}}{B_{\ell}-Q_{\ell}} B_{\ell}^{-1} \sum_{s \in \Omega} \frac{p_{s}}{k_{\ell+1, s}} L^{b_{\ell, s}} \\
& +\sum_{m=1}^{N-1} \gamma_{m} \frac{Q_{m}}{B_{\ell}-Q_{\ell}} \sum_{s \in \Omega}\left(\frac{p_{s}}{k_{\ell, s}} L^{a_{\ell, s}}-\frac{p_{s}}{k_{\ell+1, s}} L^{b_{\ell, s}}\right) \\
= & \sum_{m=1}^{N-1} \gamma_{m} \sum_{s \in \Omega}\left(\frac{p_{s}}{k_{m, s}} L^{a_{m, s}}-\frac{p_{s}}{k_{m+1, s}} L^{b_{m, s}}\right)+\sum_{m=1}^{N-1} \gamma_{m} \frac{Q_{m}}{B_{\ell}-Q_{\ell}} \sum_{s \in \Omega} \frac{p_{s}}{k_{\ell, s}} L^{a_{\ell, s} .}
\end{aligned}
$$

By (33) this implies that $h_{\gamma}=\hat{h}_{\hat{\gamma}}$.

If the partitions $\left\{I_{n}\right\}_{1 \leq n \leq N}$ and $\left\{\hat{I}_{n}\right\}_{1 \leq n \leq N}$ differ in more than one partition point $z_{\ell}$, we can obtain the results from the above by changing one partition point at a time. 
The next lemma states that adding extra points to the set $z_{0}, \ldots, z_{N}$ does not influence the set of densities obtained from Theorem 4.1. This lemma is one of the ingredients of the proof of Theorem 5.3 below.

LEMMA 5.2. Let $T$ be a random system with partition $\left\{I_{i}\right\}_{1 \leq i \leq N}$ and corresponding partition points $z_{0}, \ldots, z_{N}$. Consider a refinement of the partition, given by adding extra points $z_{1}^{\dagger}, \ldots, z_{s}^{\dagger}$, for some $s \in \mathbb{N}$. Let $T^{\dagger}$ be the corresponding random system, that is, $T^{\dagger}(x)=T(x)$ for all $x \in[0,1]$, and let $M^{\dagger}$ be the fundamental matrix of $T^{\dagger}$. There is a one-to-one correspondence between the solutions $\gamma$ of $M \gamma=0$ and the solutions $\gamma^{\dagger}$ of $M^{\dagger} \gamma^{\dagger}=0$. Moreover, the functions $h_{\gamma}$ and $h_{\gamma^{\dagger}}^{\dagger}$ coincide.

Proof. Let $Z^{\dagger}:=\left\{z_{1}^{\dagger}, \ldots, z_{s}^{\dagger}\right\}$. By introducing these extra points the fundamental matrix $M^{\dagger}$ of $T^{\dagger}$ becomes an $(N+s) \times(N+s-1)$ matrix. It is possible to construct this matrix from $M$ in $s$,

$$
M \rightarrow M_{1}^{\dagger} \rightarrow M_{2}^{\dagger} \rightarrow \cdots \rightarrow M_{s}^{\dagger}=M^{\dagger}
$$

by adding one of the points from $Z^{\dagger}$ to the partition of $T$ at a time. All of these steps work in exactly the same way, so it is enough to prove the result for $s=1$. Therefore, assume $Z^{\dagger}=\left\{z^{\dagger}\right\}$. There is an $1 \leq i \leq N$ such that $z^{\dagger}$ splits the interval $I_{i}$ into two subintervals, say $I_{i}^{L}$ and $I_{i}^{R}$. By Proposition 5.1, it is irrelevant whether $z^{\dagger} \in I_{i}^{L}$ or $z^{\dagger} \in I_{i}^{R}$. By construction, $z^{\dagger}$ is a continuity point of $T^{\dagger}=T$, so

$$
a_{i, j}^{\dagger}=b_{i, j}^{\dagger}=k_{i, j} z^{\dagger}+d_{i, j}
$$

and for each $n$ we have

$$
\sum_{j \in \Omega}\left[\frac{p_{j}}{k_{i, j}} \mathrm{KI}_{n}\left(a_{i, j}^{\dagger}\right)-\frac{p_{j}}{k_{i, j}} \mathrm{KI}_{n}\left(b_{i, j}^{\dagger}\right)\right]=0 .
$$

Therefore $M^{\dagger}$ has, with respect to $M$, an extra column at the $i$ th position, whose entries are all zeros except for the diagonal and subdiagonal entries, which are given by $\sum_{j \in \Omega}\left(p_{j} / k_{i, j}\right)$ and $-\sum_{j \in \Omega}\left(p_{j} / k_{i, j}\right)$, respectively. Moreover, the $i$ th and $(i+1)$ th rows of $M^{\dagger}$ are obtained by splitting the $i$ th row of $M$ into two, such that $\mathrm{KI}_{i}\left(a_{n, j}\right)=$ $\mathrm{KI}_{i}^{\dagger}\left(a_{n, j}\right)+\mathrm{KI}_{i+1}^{\dagger}\left(a_{n, j}\right)$ for all $n$, and analogously for $b_{n, j}$.

The null space of $M^{\dagger}$ equals the null space of the $(N+1) \times N$ matrix $A$ obtained from $M^{\dagger}$ by replacing the $(i+1)$ th row by the sum of the $i$ th and $(i+1)$ th rows. Then all the entries of the $i$ th column of $A$ are 0 except for the diagonal entry, and the matrix $M$ appears as a submatrix of $A$, by deleting the $i$ th column and the $i$ th row. Hence, any solution $\gamma$ of $M \gamma=0$ can be transformed into a solution $\gamma^{\dagger}$ of $M^{\dagger} \gamma^{\dagger}=0$ by setting $\gamma_{j}^{\dagger}=\gamma_{j}$ for $j \neq i$ and by using the relation $\sum_{j=1}^{N} A_{i, j} \gamma_{j}^{\dagger}=0$ for $\gamma_{i}^{\dagger}$. This gives the first part of the lemma.

Finally, for corresponding solutions $\gamma$ and $\gamma^{\dagger}$ the associated densities $h_{\gamma}$ and $h_{\gamma^{\dagger}}^{\dagger}$ coincide, since

$$
\sum_{j \in \Omega}\left[\frac{p_{j}}{k_{i, j}} L_{a_{i, j}^{\dagger}}(x)-\frac{p_{j}}{k_{i, j}} L_{b_{i, j}^{\dagger}}(x)\right]=0 .
$$


The next theorem says that in the case where all maps $T_{j}$ are expanding, Theorem 4.1 in fact produces all absolutely continuous invariant measures for the system $T$.

THEOREM 5.3. Let $\Omega \subseteq \mathbb{N}$ and let $T$ be a random piecewise linear system satisfying assumptions (A1), (A3), (A4) and (A5). Furthermore, assume that $\left|k_{i, j}\right|>1$ for each $j \in \Omega$ and $1 \leq i \leq N$. An $L^{1}(\lambda)$-function $h$ is an invariant function for the random system $T$ if and only if $h=h_{\gamma}$ for some solution $\gamma$ of the system $M \gamma=0$.

An essential ingredient in the proof of this theorem is the extension of a result by Boyarksy, Góra and Islam from [GBI06] given in the next lemma. [GBI06, Theorem 3.6] states that if we have a random system consisting of two maps that are both expanding, the supports of the invariant densities of $T$ are a finite union of intervals. As the next lemma shows, this result in fact goes through for any finite or countable number of maps with only a small change in the proof. In the case of piecewise linear maps, some small steps can be slightly simplified. We have included the proof for the convenience of the reader.

LemmA 5.4. (cf. Lemma 3.4 and Theorem 3.6 from [GBI06]) Let $\Omega \subseteq \mathbb{N}$ and let $T$ be a random system of piecewise linear maps satisfying (A1) and such that for each $j \in \Omega$ the map $T_{j}$ is expanding, that is, it satisfies $\left|k_{i, j}\right|>1$ for all $1 \leq i \leq N$. If $h$ is a $T$-invariant density, then the support of $h$ is a finite union of open intervals.

Proof. Let $H=\left\{v_{1}, \ldots, v_{r}\right\}$ be the base of the subspace of $L^{1}(\lambda)$ of $T$-invariant functions, consisting of density functions of bounded variation, mentioned in $\S 2$. Since any invariant function $h$ for $T$ can be written as $h=\sum_{n=1}^{r} c_{n} v_{n}$ for some constants $c_{n} \in \mathbb{R}$, it is enough to prove the result for elements in $H$. Therefore, let $h \in H$ and let $U:=\operatorname{supp}(h)$ denote the support of $h$. Since $h$ is a function of bounded variation, we can take $h$ to be lower semicontinuous and $U$ can be written as a countable union of open intervals, each separated by an interval of positive length: $U=\bigcup_{k \geq 1} U_{k}$. Assume without loss of generality that $\lambda\left(U_{k+1}\right) \leq \lambda\left(U_{k}\right)$ for each $k \geq 1$. Let $Z:=\left\{z_{1}, \ldots, z_{N-1}\right\}$ and let $\mathcal{D}$ be the set of indices $k$, such that $U_{k}$ contains one of the points $z \in Z$, that is,

$$
\mathcal{D}=\left\{k \geq 1 \mid \exists z \in Z: z \in U_{k}\right\}
$$

We first show that $\mathcal{D} \neq \varnothing$ by proving that $Z \cap U_{1} \neq \emptyset$. Suppose to the contrary that $U_{1}$ does not contain a point $z$. Then for each $j \in \Omega, T_{j}\left(U_{1}\right)$ is an interval and since each $T_{j}$ is expanding, we have $\lambda\left(T_{j}\left(U_{1}\right)\right)>\lambda\left(U_{1}\right)$. By the property from (3) that $U$ is forward invariant, we know that $T_{j}\left(U_{1}\right) \subseteq U$ for each $j$, so it must be contained in one of the intervals $U_{k}$. This gives a contradiction.

Now, let $J$ be the smallest interval in the set

$$
\left\{U_{k} \cap I_{n}: k \in \mathcal{D}, 1 \leq n \leq N\right\} .
$$

Note that this is a finite set, since $Z$ and $\mathcal{D}$ are both finite. Moreover, by the above this set is not empty, so $J$ exists. Since each $U_{k}$ is an open interval, we have $\lambda(J)>0$. Let $\mathcal{F}=$ $\left\{k \geq 1: \lambda\left(U_{k}\right) \geq \lambda(J)\right\}$, where $k$ is not necessarily in $J$, and let $S=\bigcup_{k \in \mathcal{F}} U_{k}$. Since any connected component $U_{k}$ of $S$ has Lebesgue measure bigger than $\lambda(J), S$ is a finite union of open intervals. We first prove that $T_{j}(S) \subseteq S$ for any $j \in \Omega$. Let $U_{k} \subseteq S$ and suppose 
first that $k \notin \mathcal{D}$. Then, for each $j \in \Omega$, as above $T_{j}\left(U_{k}\right)$ is an interval with $\lambda\left(T_{j}\left(U_{k}\right)\right)>$ $\lambda\left(U_{k}\right) \geq \lambda(J)$. So, $T_{j}\left(U_{k}\right)$ is contained in another interval $U_{i}$ that satisfies $\lambda\left(U_{i}\right)>\lambda(J)$ and thus satisfies $U_{i} \subseteq S$. Hence, $T_{j}\left(U_{k}\right) \subseteq S$. If, on the other hand, $k \in \mathcal{D}$, then $T_{j}\left(U_{k}\right)$ consists of a finite union of intervals and since $T_{j}$ is expanding, the Lebesgue measure of each of these intervals exceeds $\lambda(J)$. Hence, each of the connected components of $T_{j}\left(U_{k}\right)$ is contained in some interval $U_{i}$ that satisfies $\lambda\left(U_{i}\right)>\lambda(J)$ and therefore $U_{i} \subseteq S$. Hence, also in this case $T_{j}\left(U_{k}\right) \subseteq S$, implying that $T_{j}(S) \subseteq S$ for all $j \in \Omega$.

Obviously, $S \subseteq U$. Using the fact that $T_{j}(S) \subseteq S$ for all $j \in \Omega$, we will now show that $U \subseteq S$. Suppose this is not the case and let $U_{s}$ be the largest interval in $U \backslash S$. Since $U_{k} \subseteq$ $S$ for any $k \in \mathcal{D}$, we have $s \notin \mathcal{D}$. So, again, for each $j \in \Omega$ the set $T_{j}\left(U_{s}\right)$ is an interval with $\lambda\left(T_{j}\left(U_{s}\right)\right)>\lambda\left(U_{s}\right)$ and hence, $T_{j}\left(U_{s}\right) \subseteq S$. Thus $U_{s} \subseteq T_{j}^{-1}(S)$ and since $U_{s} \nsubseteq S$, we have $U_{s} \subseteq T_{j}^{-1}(S) \backslash S$. Let $\mu_{\mathbf{p}}$ be the absolutely continuous $T$-invariant measure with density $h$. We show that $\mu_{\mathbf{p}}\left(T_{j}^{-1}(S) \backslash S\right)=0$. Since for each $j \in \Omega$ we have

$$
S \subseteq T_{j}^{-1}\left(T_{j}(S)\right) \subseteq T_{j}^{-1}(S),
$$

we obtain from (1) that

$$
\begin{aligned}
0 & =\mu_{\mathbf{p}}(S)-\mu_{\mathbf{p}}(S)=\sum_{j \in \Omega} p_{j} \mu_{\mathbf{p}}\left(T_{j}^{-1}(S)\right)-\sum_{j \in \Omega} p_{j} \mu_{\mathbf{p}}(S) \\
& =\sum_{j \in \Omega} p_{j}\left(\mu_{\mathbf{p}}\left(T_{j}^{-1}(S)\right)-\mu_{\mathbf{p}}(S)\right)=\sum_{j \in \Omega} p_{j} \mu_{\mathbf{p}}\left(T_{j}^{-1}(S) \backslash S\right) .
\end{aligned}
$$

Since $p_{j}>0$ for all $j$, we have that $\mu_{\mathbf{p}}\left(T_{j}^{-1}(S) \backslash S\right)=0$ for each $j$. Hence, $\mu_{\mathbf{p}}\left(U_{s}\right)=0$, which contradicts the fact that $U_{s} \subseteq U$.

Remark 5.5. The paper [GBI06] contains an example that shows that the previous lemma is not necessarily true if we drop the assumption that all maps $T_{j}$ are expanding. In [GBI06, Example 3.7] the authors describe a random system $T$ using an expanding and a non-expanding map, of which for a certain probability vector $\mathbf{p}$ the support of the invariant density is a countable union of intervals. The fact that the supports of the elements from $H$ are finite unions of open intervals plays an essential role in the proof of Theorem 5.3, as we will now show.

Proof of Theorem 5.3. We will show that the linear mapping from the null space of $M$ to the subspace of $L^{1}(\lambda)$ of all $T$-invariant functions is a linear isomorphism. Let $H=\left\{v_{1}, \ldots, v_{r}\right\}$ again be the basis of density functions of bounded variation, whose corresponding measures are ergodic, for the subspace of $T$-invariant $L^{1}(\lambda)$-functions mentioned in $\S 2$. Recall that any invariant function $h$ for $T$ can be written as $h=$ $\sum_{n=1}^{r} c_{n} v_{n}$ for some constants $c_{n} \in \mathbb{R}$.

The injectivity follows from the proof of Theorem 4.1, where we showed that $h_{\gamma}=0$ implies $\gamma=0$. We prove surjectivity by providing for each $h \in H$ a vector $\gamma$ such that $h_{\gamma}=h$. We will do this by altering $T$ in several steps (see Figure 2), so that we finally obtain a system $T_{U}$ that has a vector $\gamma_{U}$ associated to it for which the corresponding density $h_{\gamma_{U}}^{U}$ vanishes outside the support $U$ of $h$. Then, using Proposition 5.1 and Lemma 5.2, we transform the solution $\gamma_{U}$ to a solution $\gamma$ for $T$ that produces the original density $h$. 


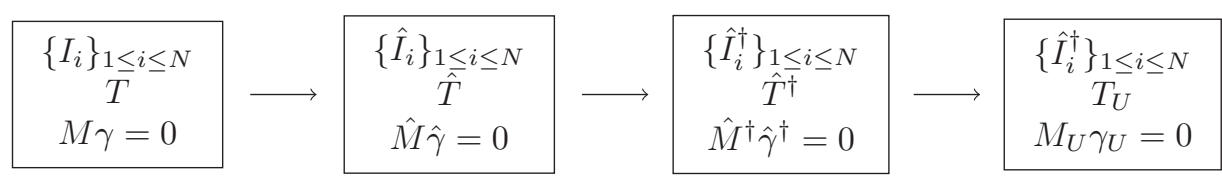

FIGURE 2. The steps we take in transforming $T$ to $T_{U}$.

Fix $h \in H$, and let $U:=\operatorname{supp}(h)$. Let $Z=\left\{z_{1}, \ldots, z_{N-1}\right\}$ again be the set of critical points of the system. Following [Kop90, Theorem 2], we classify the points in $Z$ as follows:

$Z_{1}:=\left\{z_{i} \in Z \mid z_{i}\right.$ is in the interior of $\left.U\right\}$,

$Z_{2}:=\left\{z_{i} \in Z \mid z_{i}\right.$ is a left (right) endpoint of a subinterval of $U$ and $\left.z_{i} \in I_{i+1}\left(z_{i} \in I_{i}\right)\right\}$,

$Z_{3}:=\left\{z_{i} \in Z \mid z_{i}\right.$ is a left (right) endpoint of a subinterval of $U$ and $\left.z_{i} \in I_{i}\left(z_{i} \in I_{i+1}\right)\right\}$,

$Z_{4}:=\left\{z_{i} \in Z \mid z_{i}\right.$ is an exterior point for $\left.U\right\}$.

We now modify the partition $\left\{I_{i}\right\}_{1 \leq i \leq N}$ on the points in $Z_{3}$, so that it corresponds better to the set $U$. Let $\left\{\hat{I}_{i}\right\}_{1 \leq i \leq N}$ be a partition of $[0,1]$ given by $z_{0}, \ldots, z_{N}$ and differing from $\left\{I_{i}\right\}_{1 \leq i \leq N}$ only for $z_{i} \in Z_{3}$, that is, $z_{i} \in \hat{I}_{i}$ if and only if $z_{i} \notin I_{i}$. Let $\hat{T}$ be the corresponding random system, that is, $\hat{T}(x)=T(x)$ for all $x \notin Z_{3}$. By Proposition 5.1, the corresponding matrices $M$ and $\hat{M}$ have vectors in their null spaces that differ only on the entries $i$ for which $z_{i} \in Z_{3}$, but such that they define the same density.

There might be boundary points of $U$ that are not in $Z$. Let $Z^{\dagger}$ be the set of such points. From Lemma 5.4 it follows that $U$ is a finite union of open intervals, so the set $Z^{\dagger}$ is finite. Consider the partition $\left\{\hat{I}_{i}^{\dagger}\right\}$ given by the points in $Z \cup Z^{\dagger}$ and let $\hat{T}^{\dagger}$ be the system with this partition and given by $\hat{T}^{\dagger}(x)=\hat{T}(x)$ for all $x$. By Lemma 5.2, the corresponding matrices $\hat{M}$ and $\hat{M}^{\dagger}$ have vectors in their null spaces that differ only on the extra entries corresponding to points $z^{\dagger} \in Z^{\dagger}$, but such that they define the same density.

Define a new piecewise linear random system $T_{U}$ by modifying $\hat{T}^{\dagger}$ outside of $U$. To be more precise, we let $T_{U}(x)=\hat{T}^{\dagger}(x)$ for all $x \in U$ and on each connected component of $[0,1] \backslash U$ we assume all maps $T_{U, j}$ to be equal and onto, that is, mapping the interval onto $[0,1]$. Recall from (3) that the set $U$ is forward invariant under $T$. Then any invariant function of $T_{U}$ vanishes on $[0,1] \backslash U \lambda$-a.e., since the set of points $x \in[0,1] \backslash U$, such that $T^{n}(x) \in[0,1] \backslash U$ for all $n \geq 0$ is a self-similar set of Hausdorff dimension less than 1. From Theorem 4.1 we get a non-trivial solution $\gamma_{U}$ of $M_{U} \gamma_{U}=0$ with a corresponding function $h_{U}$ that vanishes on $[0,1] \backslash U$. Since $\hat{T}$ and $T_{U}$ coincide on $U$, the function $h_{U}$ is also invariant for $\hat{T}$ and hence for $T$. From the fact that $U$ is the support of one of the densities in the basis $H$ and $\operatorname{supp}\left(h_{U}\right) \subseteq U$, we then conclude that $h_{U}=h$, up to possibly a set of Lebesgue measure 0 .

It remains to show that $\gamma_{U}$ can be transformed into a vector from the null space of $M$, leading to the same density $h_{U}$. We first show that $\hat{M}^{\dagger} \gamma_{U}=0$. Note that for $z_{i} \in Z_{4}$, since $h_{U}$ is of bounded variation,

$$
\lim _{x \uparrow z_{i}} h_{U}(x)=0=\lim _{x \downarrow z_{i}} h_{U}(x) .
$$


Hence, by the calculations in (25), $\gamma_{U, i}=0$. Similarly, for $z_{i} \in Z_{2} \cup Z_{3}$ we have that either $\lim _{x \uparrow z_{i}} h_{U}(x)=0$ or $\lim _{x \downarrow z_{i}} h_{U}(x)=0$, which again by the calculations in (25) gives $\gamma_{U, i}=0$. Hence, $\gamma_{U, i}=0$ for each $i$ such that $z_{i} \in Z_{2} \cup Z_{3} \cup Z_{4}$. Similarly, $\gamma_{U, i}=0$ for each $i$ such that $z_{i} \in Z^{\dagger}$. In the multiplication $\hat{M}^{\dagger} \gamma_{U}$ the orbits of the points $a_{i, j}$ and $b_{i, j}$ which are different under $\hat{T}^{\dagger}$ and $T_{U}$ are multiplied by 0 . Since $U$ is forward invariant, all orbits of points $a_{i, j}$ and $b_{i, j}$ corresponding to $i$ such that $z_{i} \in Z_{1}$ will stay in $U$ and will thus be equal under $\hat{T}^{\dagger}$ and $T_{U}$. These facts imply that also $\hat{M}^{\dagger} \gamma_{U}=0$ and that the corresponding invariant density for $\hat{T}^{\dagger}$ is again $h_{U}$.

From Lemma 5.2 it follows that there is a vector $\hat{\gamma}$ in the null space of $\hat{M}$ with $\hat{h}_{\hat{\gamma}}=h_{U}$. Finally, Proposition 5.1 then tells us how we can modify $\hat{\gamma}$ to get a vector $\gamma$ in the null space of $M$ with $h_{\gamma}=\hat{h}_{\hat{\gamma}}=h_{U}=h$.

\section{Examples}

In this section we apply Theorems 4.1 and 5.3 to various examples.

6.1. Random tent maps. For any countable set of slopes $\left\{k_{j}\right\}_{j \in \Omega}$ with $k_{j} \in(0,2)$ for each $j$, consider the family $T:=\left\{T_{j}\right\}_{j \in \Omega}$, where each $T_{j}$ is a tent map of slope $k_{j}$, that is, $T_{j}:[0,1] \rightarrow[0,1]$ is given by

$$
T_{j}(x)= \begin{cases}k_{j} x & \text { if } x \in[0,1 / 2], \\ k_{j}-k_{j} x & \text { if } x \in(1 / 2,1]\end{cases}
$$

(see Figure 3(a)). So, (A1) and (A4) hold.

Let $\mathbf{p}=\left(p_{j}\right)_{j \geq 0}$ be a probability vector such that $T$ is expanding on average (i.e. $\left.\sum_{j \in \mathbb{N}}\left(p_{j} / k_{j}\right)<1\right)$, so (A2) holds. One easily verifies that then conditions (A3) and (A5) hold as well. For $N=2$ set

$$
z_{0}=0, \quad z_{1}=\frac{1}{2}, \quad z_{2}=1,
$$

and $I_{1}=\left[z_{0}, z_{1}\right], I_{2}=\left(z_{1}, z_{2}\right]$. Since $z_{1}$ is the only critical point, the fundamental matrix $M$ is the null vector. As a consequence, we can choose $\gamma=1$, to obtain the invariant density

$$
h_{\gamma}=c \sum_{j \in \Omega} \frac{2 p_{j}}{k_{j}} L_{k_{j} / 2},
$$

for some normalizing constant $c$. If, for each $j \in \mathbb{N}$ and $w \in \Omega^{t}, t \geq 0$, we set

$$
\ell_{\omega, j}=\#\left\{1 \leq n \leq t: T_{\omega_{1}^{n-1}}\left(\frac{k_{j}}{2}\right) \in\left(\frac{1}{2}, 1\right]\right\},
$$

then this becomes

$$
h_{\gamma}=c \sum_{j \in \Omega} \frac{2 p_{j}}{k_{j}} \sum_{t \geq 0} \sum_{\omega \in \Omega^{t}}(-1)^{\ell_{\omega, j}} \prod_{n=0}^{t} \frac{p_{\omega_{n}}}{k_{\omega_{n}}} \mathbf{1}_{\left[0, T_{\omega}\left(k_{j} / 2\right)\right)} .
$$

If we assume that $k_{j}>1$ for all $j$, then it follows from Theorem 5.3 that the density from (36) is the unique invariant density for $(T, \mathbf{p})$. If we do not assume this, then we can still 


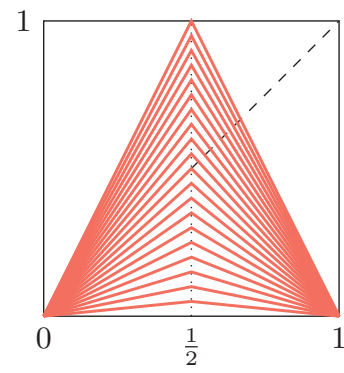

(a) Countably many tent maps.

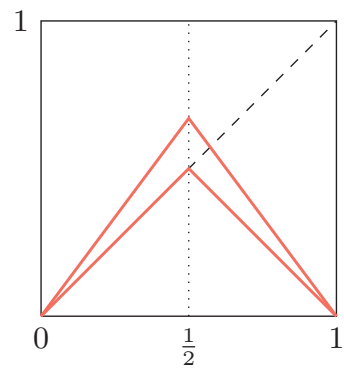

(b) Two tent maps.

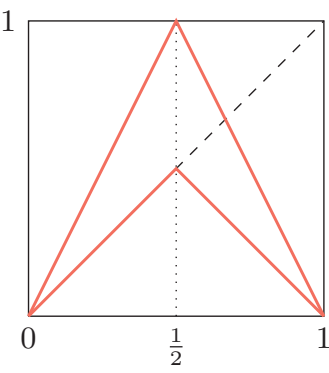

(c) Linear logistic maps.

FIGURE 3. Random families of tent maps.

draw the same conclusion if there are only finitely many maps. Namely, to satisfy condition (A2) there has to be at least one $j$ such that $k_{j}>1$. The existence and uniqueness of an absolutely continuous invariant measure for the map $T_{j}$ are then guaranteed by the results from [LY73, LY78]. If the set $\left\{k_{j}\right\}_{j \in \mathbb{N}}$ is finite, it then follows from [Pel84, Corollary 7] that there is only one invariant density for $(T, \mathbf{p})$.

In [AGH18] the authors considered random combinations of logistic maps. In [AGH18, Theorem 4.2] they proved that the random system $\left\{f_{0}, f_{1}\right\}$ with $f_{0}(x)=2 x(1-x)$ and $f_{1}(x)=4 x(1-x)$ has a $\sigma$-finite absolutely continuous invariant measure that is infinite if the map $f_{0}$ is chosen with probability $p_{0}>\frac{1}{2}$. The linear analogue of this system paints a different picture. Fix $a \in(1,2]$ and consider the random system with two maps $T_{0}(x)=\min \{x, 1-x\}$ and $T_{a, 1}(x)=\min \{a x, a-a x\}$. See Figure 3(b) for an example with $a=\frac{4}{3}$. For any $p \in(0,1)$, set $p_{0}=p$ and $p_{1}=1-p$ and note that $p_{0}+p_{1} / a<1$. Assumptions (A1)-(A5) are then met and the random system $T=\left\{T_{0}, T_{a, 1}\right\}$ has a finite absolutely continuous invariant measure for any such $p$. A straightforward computation yields $L_{1 / 2}=(1 /(1-p)) \mathbf{1}_{[0,1 / 2)}+(1 / a) L_{a / 2}$, so that up to a normalizing constant, the unique absolutely continuous invariant density is then

$$
h_{\gamma, a}=\frac{2 p}{1-p} \mathbf{1}_{[0,1 / 2)}+\frac{2}{a} L_{a / 2} .
$$

In particular, for $a=2$ as shown in Figure 3(c) we get

$$
h_{\gamma, 2}=(1+p) \mathbf{1}_{[0,1 / 2]}+(1-p) \mathbf{1}_{(1 / 2,1]} .
$$

Note that for $p=1$ we have a deterministic, non-expanding interval map that does not satisfy the requirements from [Kop90]. However, the $\operatorname{limit}_{\lim _{p \rightarrow 1}} h_{\gamma, 2}=2 \cdot \mathbf{1}_{[0,1 / 2]}$ is an invariant density for the system. On the other hand, for a fixed $p \in(0,1)$ the limit $\lim _{a \rightarrow 1} h_{\gamma, a}$ is not an absolutely continuous measure. To see this, note that $h_{\gamma, a}$ is determined by the random orbits of $a / 2$ and that $1-a / 2 \leq T_{\omega}(a / 2) \leq a / 2$ for any $\omega$. Hence, by (37) and the definition of the $L$-functions in (17), it follows that $h_{\gamma, a}=0$ on $(a / 2,1]$, while on $[0,1-a / 2)$ we have $h_{\gamma, a}=v$ on $[0,1-a / 2)$ for some constant $v \in \mathbb{R}$. For any point in $x \in[0,1-(a / 2))$, the random Perron-Frobenius operator from (2) 
now yields

$$
v=h_{\gamma, a}(x)=P_{T} h_{\gamma, a}(x)=p v+(1-p) \frac{v}{a},
$$

which holds if and only if $v=0$. It follows that for any $a \in(1,2]$ and any $p \in(0,1)$, $\operatorname{supp}\left(h_{\gamma, a}\right) \subseteq[1-a / 2, a / 2]$. As a consequence $\lim _{a \rightarrow 1} h_{\gamma}=\delta_{1 / 2}$, where $\delta_{1 / 2}$ is the Dirac delta function at $\frac{1}{2}$.

6.2. A random family of $W$-shaped maps. Keller introduced in [Kel82] a family of piecewise expanding $W$-maps to study the phenomenon of instability of absolutely continuous invariant measures. Later the stability of $W$-shaped maps was studied in other papers as well; see, for example, [LGB +13, EM12]. Here we construct a random family of $W$-shaped maps, where each element of the collection is an expanding on average random map $W_{a}:=\left\{W_{a, 0}, W_{a, 1}\right\}$ defined on the unit interval. We give an absolutely continuous invariant probability measure.

For $a>2$, let $\Omega=\{0,1\}$ and $N=4$. Set

$$
z_{0}=0, \quad z_{1}=1 / a, \quad z_{2}=1 / 2, \quad z_{3}=(a-1) / a, \quad z_{4}=1
$$

and

$$
I_{1}=\left[z_{0}, z_{1}\right], \quad I_{2}=\left(z_{1}, z_{2}\right], \quad I_{3}=\left(z_{2}, z_{3}\right), \quad I_{4}=\left[z_{3}, z_{4}\right]
$$

Let

$$
W_{a, 0}(x)= \begin{cases}1-a x & \text { if } x \in I_{1}, \\ \frac{2}{a-2} x-\frac{2}{(a-2) a} & \text { if } x \in I_{2}, \\ W_{a, 0}(1-x) & \text { otherwise }\end{cases}
$$

and

$$
W_{a, 1}(x)= \begin{cases}1-a x & \text { if } x \in I_{1}, \\ \frac{2(a-1)}{a-2} x-\frac{2(a-1)}{(a-2) a} & \text { if } x \in I_{2}, \\ W_{a, 1}(1-x) & \text { otherwise. }\end{cases}
$$

See Figure 4(a), for some examples of $W_{a}$ for different values of $a$.

For $a>4$ the map $W_{a, 0}$ presents two contractive branches. Let $1>p>((a-4)(a-1)) /$ $(a-2)^{2}$ be arbitrary, and let $p_{a, 0}=1-p$ and $p_{a, 1}=p$. With this choice of probability vector the random map $W_{a}$ satisfies (A1)-(A5). The fundamental matrix $M$ is given by

$$
M=\left(\begin{array}{ccc}
\frac{1-a}{a^{2}}-\frac{C}{a} & \frac{p_{a 0}(2-a)(a-1)}{a^{2}}+\frac{p_{a 1}(2-a)}{a^{2}(a-1)} & -\frac{C}{a}+\frac{1}{a^{2}} \\
C & -C & 0 \\
0 & -C & C \\
\frac{1}{a^{2}(a-1)}-\frac{C}{a(a-1)} & \frac{p_{a 0}(2-a)}{a^{2}(a-1)}-\frac{p_{a 1}(2-a)\left(a^{2}-a-1\right)}{a^{2}(a-1)^{2}} & -\frac{C}{a(a-1)}+\frac{1+a-a^{2}}{a^{2}(a-1)}
\end{array}\right)
$$

for some constant $C$. Its null space consists of all vectors of the form

$$
s\left(\begin{array}{lll}
1 & 1 & 1
\end{array}\right)^{\top}, \quad s \in \mathbb{R} .
$$




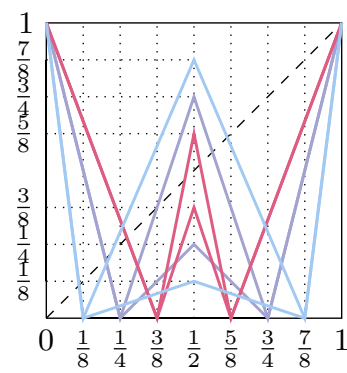

(a) Examples of $W_{a}$ for $a=8,4, \frac{8}{3}$

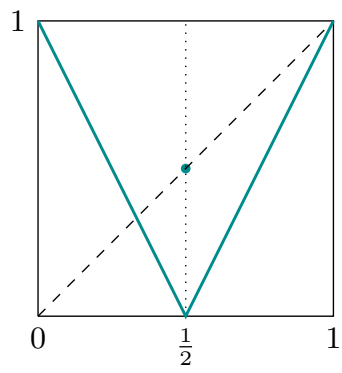

(b) $W_{2}$

FIGURE 4. Examples of random systems $W_{a}$ for various values of $a$.

From

$L_{0}=\frac{1}{1-a}, \quad L_{1 / a}=\frac{1}{a(a-1)}+\mathbf{1}_{[0,1 / a]} \quad$ and $\quad L_{(a-1) / a}=-\frac{1}{a(a-1)}+\mathbf{1}_{[0,(a-1) / a]}$,

we get the invariant density

$h_{a, p}=c\left[((a-1)-p(a-2)) \cdot \mathbf{1}_{[0,1 / a)}+\mathbf{1}_{[1 / a,(a-1) / a]}+\left(1-p \frac{a-2}{a-1}\right) \cdot \mathbf{1}_{((a-1) / a, 1]}\right]$,

for the normalizing constant

$$
c=\frac{a(a-1)}{2(a-1)^{2}-p a(a-2)} .
$$

Theorem 5.3 implies that if $a<4$, then this is the unique absolutely continuous invariant density for $W_{a}$. Note that

$$
\lim _{a \rightarrow 2} h_{a, p}(x)=\frac{1}{2} \mathbf{1}_{[0,1]}(x)+\frac{1}{2} \delta_{1 / 2}(x) .
$$

On the other hand, for the limit map $W_{2}$ shown in Figure 4(b), Lebesgue measure is the only absolutely continuous invariant measure.

6.3. Random $\beta$-transformations. Let $\beta>1$ be a non-integer and use $\lfloor\beta\rfloor$ to denote the largest integer not exceeding $\beta$. A $\beta$-expansion of a real number $x \in[0,\lfloor\beta\rfloor /(\beta-1)]$ is an expression of the form $x=\sum_{n=1}^{\infty} b_{n} \beta^{-n}$, where $b_{n} \in\{0,1, \ldots,\lfloor\beta\rfloor\}$ for all $n \geq 1$. The properties of $\beta$-expansions have been thoroughly studied. One of the more striking results is that Lebesgue almost all $x \in[0,\lfloor\beta\rfloor /(\beta-1)]$ have uncountably many different $\beta$-expansions (see [EJK90, Sid03, DdV07]). In [DK03] Dajani and Kraaikamp introduced a random system that produces for each $x \in[0,\lfloor\beta\rfloor /(\beta-1)]$ all its possible $\beta$-expansions. We will define this system for $1<\beta<2$ for simplicity, but everything easily extends to $\beta>2$. Set

$$
z_{0}=0, \quad z_{1}=\frac{1}{\beta}, \quad z_{2}=\frac{1}{\beta(\beta-1)}, \quad z_{3}=\frac{1}{\beta-1},
$$




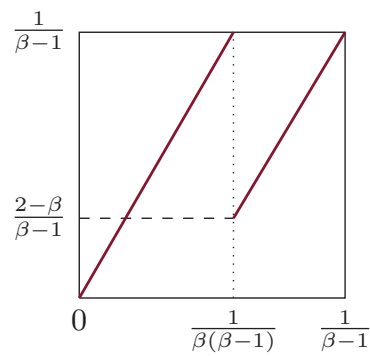

(a) $T_{0}$

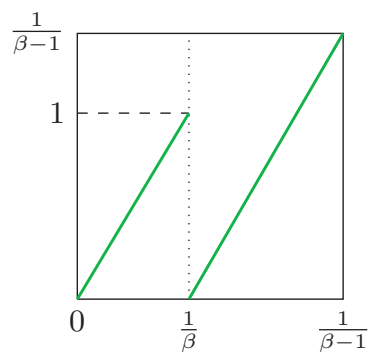

(b) $T_{1}$

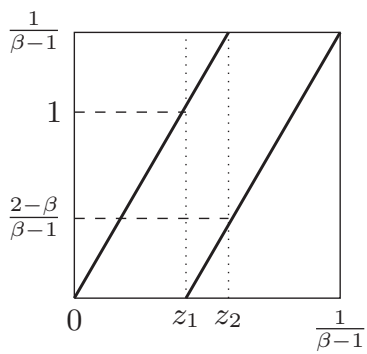

(c) $T$

FIGURE 5. In (a) we see the lazy $\beta$-transformation $T_{0}$, in (b) the greedy $\beta$-transformation $T_{1}$ and in (c) we see them combined. Whether or not $1>(2-\beta) /(\beta-1)$ depends on the chosen value of $\beta$.

and let

$$
T_{0}(x)=\left\{\begin{array}{ll}
\beta x & \text { if } x \in\left[z_{0}, z_{2}\right], \\
\beta x-1 & \text { if } x \in\left(z_{2}, z_{3}\right],
\end{array} \quad \text { and } \quad T_{1}(x)= \begin{cases}\beta x & \text { if } x \in\left[0, z_{1}\right), \\
\beta x-1 & \text { if } x \in\left[z_{1}, z_{3}\right]\end{cases}\right.
$$

(see Figure 5). The map $T_{0}$ is called the lazy $\beta$-transformation and the map $T_{1}$ is the greedy $\beta$-transformation. We do not bother to rescale the system to the unit interval $[0,1]$, since this has no effect on the computations.

One of the reasons why people are interested in the random $\beta$-transformation is for its relation to the infinite Bernoulli convolution; see [DdV05, DK13, Kem14]. The density of the absolutely continuous invariant measures has been the subject of several papers. For a special class of values $\beta$ an explicit expression for the density of $\mu_{\mathbf{p}}$ was found in [DdV07] using a Markov chain. In [Kem14] Kempton produced an explicit formula for the invariant density for all $1<\beta<2$ when $p_{0}=p_{1}=\frac{1}{2}$ by constructing a natural extension of the system. He states that there is a straightforward extension of this method to $\beta>2$. Recently Suzuki obtained a formula for the density of $\mu_{\mathbf{p}}$ for all $\beta>1$ and any $\mathbf{p}$ in [Suz19]. Since the random $\beta$-transformation satisfies assumptions (A1)-(A5) for any probability vector $\mathbf{p}=\left(p_{0}, p_{1}\right)$, we can also obtain the invariant density from Theorem 4.1. To illustrate our method we calculate the density for $\beta \in(1,2)$ and $p_{0}=p_{1}=\frac{1}{2}$.

Let $\Omega=\{0,1\}, N=3$ and set

$$
I_{1}=\left[z_{0}, z_{1}\right), \quad I_{2}=\left[z_{1}, z_{2}\right], \quad I_{3}=\left(z_{2}, z_{3}\right] .
$$

Define the left and right limits at each point of discontinuity:

$$
\begin{array}{llll}
a_{1,0}=1, & b_{1,0}=1, & a_{2,0}=\frac{1}{\beta-1}, & b_{2,0}=\frac{2-\beta}{\beta-1}, \\
a_{1,1}=1, & b_{1,1}=0, & a_{2,1}=\frac{2-\beta}{\beta-1}, & b_{2,1}=\frac{2-\beta}{\beta-1} .
\end{array}
$$

As pointed out in Remark 4.5, to determine $\gamma$ it would suffice to compute only one row of $M$, but for the sake of completeness we give $M$ below. Let $\mathrm{KI}_{n}(1)=c_{n}$. By the symmetry of the system, for each $x \in\left[z_{0}, z_{3}\right]$ and all $(i, j) \in\{1,2,3\} \times\{0,1\}$,

$$
T_{i, j}\left(z_{3}-x\right)=z_{3}-T_{4-i, 1-j}(x) .
$$


If for any $\omega=\omega_{1} \cdots \omega_{t} \in\{0,1\}^{*}$, we let $\bar{\omega} \in\{0,1\}^{*}$ denote the string $\bar{\omega}=(1-$ $\left.\omega_{1}\right) \cdots\left(1-\omega_{t}\right)$, then (38) implies that $T_{\omega}(1) \in I_{n}$ if and only if $T_{\bar{\omega}}((2-\beta) /(\beta-1)) \in$ $I_{4-n}$ and so $\mathrm{KI}_{n}((2-\beta) /(\beta-1))=c_{4-n}$. We obtain

$$
M=\left(\begin{array}{cc}
\frac{1}{\beta}+\frac{1}{2 \beta}\left(c_{1}-\frac{1}{\beta-1}\right) & -\frac{1}{2 \beta} c_{3} \\
-\frac{1}{\beta}+\frac{1}{2 \beta} c_{2} & \frac{1}{\beta}-\frac{1}{2 \beta} c_{2} \\
\frac{1}{2 \beta} c_{3} & -\frac{1}{\beta}-\frac{1}{2 \beta}\left(c_{1}-\frac{1}{\beta-1}\right)
\end{array}\right) .
$$

The null space consists of all vectors of the form

$$
s\left(\begin{array}{ll}
1 & 1
\end{array}\right)^{\top}, \quad s \in \mathbb{R} .
$$

From Theorem 5.3 we then know that the system $T$ has a unique invariant density. We obtain

$$
h_{\gamma}=\frac{c}{2 \beta} \sum_{t \geq 0} \sum_{\omega \in\{0,1\}^{t}}\left(\frac{1}{2 \beta}\right)^{t}\left(\mathbf{1}_{\left[0, T_{\omega}(1)\right)}+\mathbf{1}_{\left[T_{\omega}((2-\beta) /(\beta-1)), 1 /(\beta-1)\right]}\right),
$$

for some normalizing constant $c$. This matches the density found in [Kem14, Theorem 2.1] except for possibly countably many points.

If we set $p_{0} \neq \frac{1}{2}$, the computations are less straightforward. Nevertheless, we can obtain a nice closed formula for the density in specific instances. Let $p_{0}=p \in[0,1]$ be arbitrary and consider $\beta=(1+\sqrt{5}) / 2$, the golden mean. Then $\beta$ satisfies $\beta^{2}-\beta-1=0$ and the system has the nice property that $T_{2,0}\left(z_{1}\right)=z_{2}$ and $T_{2,1}\left(z_{2}\right)=z_{1}$ for $z_{1}=1 / \beta$ and $z_{2}=1$. Also note that $1 /(\beta-1)=\beta$. This specific case has also been studied in [DdV07, Example 1]. The resulting matrix $M$ is given by

$$
M=\frac{\beta}{\beta^{2}-p(1-p)}\left(\begin{array}{cc}
p^{2} & -p(1-p) \\
-p & (1-p) \\
(1-p) p & -(1-p)^{2}
\end{array}\right),
$$

and its null space consists of all vectors of the form

$$
s(1-p \quad p)^{\top}, \quad s \in \mathbb{R} .
$$

For the functions $L_{y}$ we obtain $L_{0}=0, L_{\beta}=\beta^{2}$ and

$$
\begin{aligned}
L_{1 / \beta} & =\frac{p^{2} \beta^{2}}{\beta^{2}-p(1-p)}+\frac{\beta^{2}}{\beta^{2}-p(1-p)} \mathbf{1}_{[0,1 / \beta)}+\frac{p \beta}{\beta^{2}-p(1-p)} \mathbf{1}_{[0,1)}, \\
L_{1} & =\frac{p \beta^{3}}{\beta^{2}-p(1-p)}+\frac{(1-p) \beta}{\beta^{2}-p(1-p)} \mathbf{1}_{[0,1 / \beta)}+\frac{\beta^{2}}{\beta^{2}-p(1-p)} \mathbf{1}_{[0,1)} .
\end{aligned}
$$

The unique invariant density turns out to be

$$
h_{\gamma}=\frac{\beta^{2}}{1+\beta^{2}}\left((1-p) \beta \cdot \mathbf{1}_{[0, \beta-1]}+\mathbf{1}_{(\beta-1,1)}+p \beta \cdot \mathbf{1}_{[1, \beta]}\right),
$$


which for $p=\frac{1}{2}$ corresponds to

$$
h_{\gamma}=\frac{\beta^{2}}{2\left(1+\beta^{2}\right)}\left(\beta \cdot \mathbf{1}_{[0, \beta-1]}+2 \cdot \mathbf{1}_{(\beta-1,1)}+\beta \cdot \mathbf{1}_{[1, \beta]}\right) .
$$

6.4. The random $(\alpha, \beta)$-transformation. As an example of a system that is not everywhere expanding, but is expanding on average, we consider a random combination of the greedy $\beta$-transformation and the non-expanding $(\alpha, \beta)$-transformation introduced in [DHK09]. More specifically, let $0<\alpha<1$ and $1<\beta<2$ be given and

$$
z_{0}=0, \quad z_{1}=1 / \beta, \quad z_{2}=1 .
$$

Define the $(\alpha, \beta)$-transformation $T_{0}$ on the interval $[0,1]$ by

$$
T_{0}(x)= \begin{cases}\beta x & \text { if } x \in\left[0, z_{1}\right), \\ \frac{\alpha}{\beta}(\beta x-1) & \text { if } x \in\left[z_{1}, z_{2}\right] .\end{cases}
$$

Let $T_{1}:[0,1] \rightarrow[0,1]$ be the greedy $\beta$-transformation again, given by $T_{1}(x)=\beta x$ $(\bmod 1)$. For any $0<p<(\alpha(\beta-1)) /(\beta-\alpha)$ the random system $T$ with probability vector $\mathbf{p}=(p, 1-p)$ satisfies conditions (A1), (A2), (A3) and (A5). The assumptions on the boundary points from (A4) do not hold, but this is easily solved by adding an extra interval $\left(z_{2}, z_{3}\right]$ for $z_{3}=1 /(\beta-1)$ and extending $T_{0}$ and $T_{1}$ to it by setting $T_{0}(x)=T_{1}(x)=\beta x-1$.

This random system $T$ does not satisfy the conditions of Theorem 5.3 and we cannot therefore conclude directly that Theorem 4.1 produces all invariant densities for $T$. However, the set $\Omega=\{0,1\}$ is finite and the map $T_{1}$ is expanding with $T_{1}^{\prime}(x)=\beta>1$ for all $x$ and therefore $T$ satisfies the conditions from [Pel84, Corollary 7] on the number of ergodic components of the pseudo skew-product $R$. Since the greedy $\beta$-transformation $T_{1}$ has a unique absolutely continuous invariant measure, this corollary implies that also the random system $T$ has a unique invariant density. We use Theorem 4.1 to obtain this density.

Let $0<p<(\alpha(\beta-1)) /(\beta-\alpha)$ be arbitrary and set

$$
I_{1}=\left[z_{0}, z_{1}\right), \quad I_{2}=\left[z_{1}, z_{2}\right], \quad I_{3}=\left(z_{2}, z_{3}\right] .
$$

The left and right limits at each point of discontinuity are given by

$$
\begin{array}{llll}
a_{1,0}=1, & b_{1,0}=0, & a_{2,0}=\alpha-\alpha / \beta, & b_{2,0}=\beta-1, \\
a_{1,1}=1, & b_{1,1}=0, & a_{2,1}=\beta-1, & b_{2,1}=\beta-1 .
\end{array}
$$

By construction, none of the points in $[0,1]$ will ever enter the interval $I_{3}$, therefore $\mathrm{KI}_{3}(y)=0$ for all $y \in[0,1]$. As a consequence, the last row of the $3 \times 2$ fundamental matrix $M$ is given by $\mu_{3,1}=0$ and $\mu_{3,2}=-1 / \beta$. This fact, together with the fact that we know from Lemma 3.4 that the null space of $M$ is non-trivial, forces the first column of $M$ to be zero, that is, $\mu_{1,1}=\mu_{2,1}=\mu_{3,1}=0$. Hence, the null space of $M$ consists of all vectors of the form

$$
s\left(\begin{array}{ll}
1 & 0
\end{array}\right)^{\top}, \quad s \in \mathbb{R},
$$




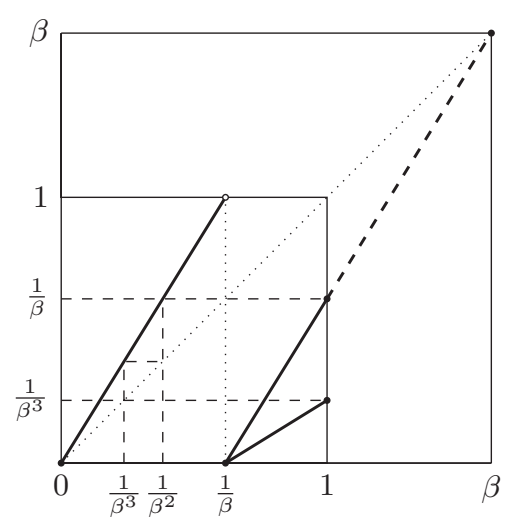

FIGURE 6. The random $(\alpha, \beta)$-transformation for $\beta=(1+\sqrt{5}) / 2$ and $\alpha=1 / \beta$.

and the unique invariant density of the system $T$ is

$$
h_{\gamma}=\frac{c}{\beta} L_{1}=\frac{c}{\beta} \sum_{t \geq 0} \sum_{\omega \in \Omega^{t}} \delta_{\omega}(1, t) \mathbf{1}_{\left[0, T_{\omega}(1)\right)},
$$

for some normalizing constant $c$. For our choice of $\beta=(1+\sqrt{5}) / 2$ and $\alpha=1 / \beta$ as in Figure 6, we can compute further to get

$$
h_{\gamma}=\frac{\beta^{2}}{\beta^{2}+1+2 p}\left(p \beta \mathbf{1}_{\left[0,1 / \beta^{3}\right]}+p \mathbf{1}_{\left[0,1 / \beta^{2}\right]}+\frac{1}{\beta} \mathbf{1}_{[0,1 / \beta]}+\mathbf{1}_{[0,1]}\right) .
$$

\section{The random Lüroth map with bounded digits}

In 1883 Lüroth introduced in [Lür83] a representation of real numbers of the unit interval, as a generalization of the decimal expansion. The standard Lüroth map on [0, 1] is defined by $T_{\mathrm{L}}(0)=0$ and

$$
T_{\mathrm{L}}(x):=n(n-1) x-(n-1) \quad \text { if } x \in\left(\frac{1}{n}, \frac{1}{n-1}\right], n \geq 2 .
$$

From $T_{\mathrm{L}}$ we can obtain the Lüroth expansion of any number $x \in(0,1]$ by assigning to it a sequence of positive integers $\left(l_{n}\right)_{n \geq 1}$, where $l_{n}$ is the unique integer such that $T_{\mathrm{L}}^{n-1}(x) \in$ $\left(\left(1 / l_{n}\right), 1 /\left(l_{n}-1\right)\right]$. The Lüroth expansion of $x$ is then the expression

$$
x=\sum_{n=1}^{\infty}\left(\left(l_{n}-1\right) \prod_{k=1}^{n} \frac{1}{l_{k}\left(l_{k}-1\right)}\right) .
$$

The map $T_{\mathrm{L}}$ was later generalized in various different ways. In [KKK90] the alternating Lüroth map was introduced as

$$
T_{\mathrm{A}}(x):=1-T_{\mathrm{L}}(x)
$$

This map is essentially a piecewise linear version of the Gauss map $x \mapsto 1 / x(\bmod 1)$, which can be used to obtain regular continued fraction expansions. This yields for each 


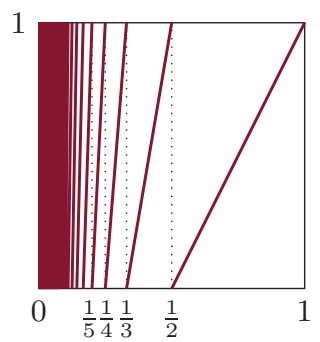

(a) $T_{L}$

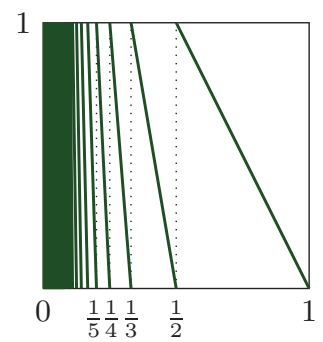

(b) $T_{A}$

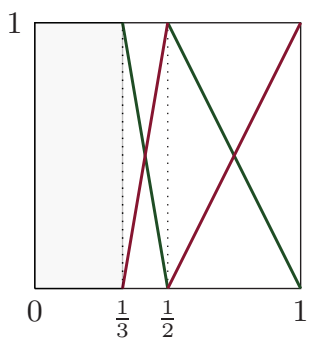

(c) $T$

FIGURE 7. In (a) we see the Lüroth map and in (b) the alternating Lüroth map. (c) shows the open random system $T$ consisting of random combinations of $T_{\mathrm{L}}$ and $T_{\mathrm{A}}$ restricted to the interval $\left[\frac{1}{3}, 1\right]$.

$x \in[0,1]$ that is not a pre-image of 0 the alternating Lüroth expansion given by

$$
x=\sum_{n=1}^{\infty}\left((-1)^{n+1} a_{n} \prod_{k=1}^{n} \frac{1}{a_{k}\left(a_{k}-1\right)}\right),
$$

where $a_{n}$ is the unique integer such that $T_{\mathrm{A}}^{n-1}(x) \in\left(1 / a_{n}, 1 /\left(a_{n}-1\right)\right]$. Further generalizations and ergodic properties of such maps were studied in [Sal68, JdV69, BBDK94] for example. In [BBDK94] it was shown among other things that among a whole family of Lüroth-type maps, the alternating Lüroth map is the one with the best approximation properties.

In this section we consider a random Lüroth map, using $T_{0}:=T_{\mathrm{L}}$ and $T_{1}:=T_{\mathrm{A}}$ as its base maps. See Figure 7 for the graphs of $T_{\mathrm{L}}, T_{\mathrm{A}}$ and an example of a random Lüroth map $T$. Then for each realization of the random system $\omega \in\{0,1\}^{\mathbb{N}}$ and each $x \in[0,1]$ that is not a pre-image of 0 under the realization $\omega$ we obtain a random Lüroth expansion by setting, for each $k \geq 0$,

$$
r_{k+1}(\omega, x)=n, \quad \text { if } T_{\omega_{1}^{k}}(x) \in\left(\frac{1}{n}, \frac{1}{n-1}\right] .
$$

Observe that

$$
T_{\omega_{1}^{k}}(x)=(-1)^{\omega_{k}} r_{k}\left(r_{k}-1\right) x+(-1)^{\omega_{k}-1}\left(r_{k}+\omega_{k}-1\right) .
$$

If we set $s_{n}=\sum_{k=1}^{n} \omega_{k}$ with $s_{0}=0$, then we obtain the following expression for $x$ :

$$
x=\sum_{n \geq 1}(-1)^{s_{n-1}}\left(r_{n}+\omega_{n}-1\right) \prod_{k=1}^{n} \frac{1}{r_{k}\left(r_{k}-1\right)} .
$$

We call this expression a random Lüroth expansion of $x$.

Many people have considered digit properties of Lüroth expansions, such as digit frequencies and the sizes of sets of numbers for which the digit sequence $\left(l_{n}\right)_{n \geq 1}$ is bounded. See for example [BI09, FLMW10, SF11, MT13, GL16]. The set of points that have all Lüroth digits bounded by some integer $a$ corresponds to the set of points that avoid the set $[0,1 / a]$ under all iterations of the map $T_{\mathrm{L}}$. For a deterministic system, such 


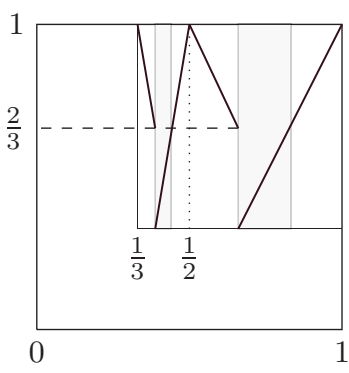

(a) $T_{0}$

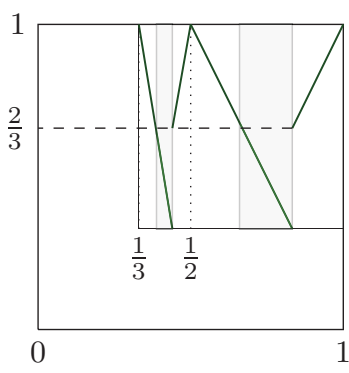

(b) $T_{1}$

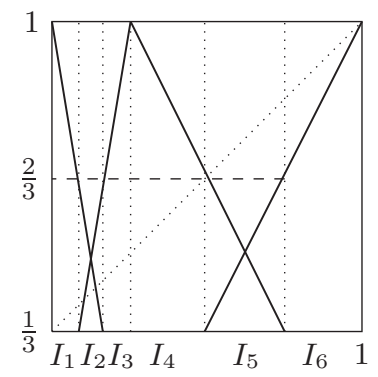

(c) $T$

FIGURE 8 . The systems $T_{0}, T_{1}$ and $T$ on the interval $I=\left[\frac{1}{3}, 1\right]$.

a set is usually a fractal no matter how large we take the upper bound $a$. In the random setting, the situation is drastically different. Fix, for example, $a=3$. We show below that all $x \in\left[\frac{1}{3}, 1\right]$ have a random Lüroth expansion using only digits 2 and 3 . Using the density given by Theorem 4.1, we can compute the frequency of each of these digits for any typical point $x \in\left[\frac{1}{3}, 1\right]$.

Partition the interval $\left[\frac{1}{3}, 1\right]$ by setting

$$
I_{1}=\left[\frac{1}{3}, \frac{7}{18}\right], I_{2}=\left(\frac{7}{18}, \frac{4}{9}\right], I_{3}=\left(\frac{4}{9}, \frac{1}{2}\right], I_{4}=\left(\frac{1}{2}, \frac{2}{3}\right], I_{5}=\left(\frac{2}{3}, \frac{5}{6}\right], I_{6}=\left(\frac{5}{6}, 1\right]
$$

Let

$$
T_{0}(x):=\left\{\begin{array}{l}
T_{\mathrm{L}}(x) \text { if } x \in I_{2} \cup I_{3} \cup I_{5} \cup I_{6}, \\
T_{\mathrm{A}}(x) \text { if } x \in I_{1} \cup I_{4},
\end{array} \text { and } T_{1}(x):=\left\{\begin{array}{l}
T_{\mathrm{A}}(x) \text { if } x \in I_{1} \cup I_{2} \cup I_{4} \cup I_{5}, \\
T_{\mathrm{L}}(x) \text { if } x \in I_{3} \cup I_{6} .
\end{array}\right.\right.
$$

For $0 \leq p \leq 1$ with $p \neq \frac{1}{2}$ let $p_{0}:=p$ and $p_{1}:=1-p$ and let $T$ now be the random Lüroth system with digits 2 and 3 defined on $\left[\frac{1}{3}, 1\right]$ by setting $T(x)=T_{j}(x)$ with probability $p_{j}$; see Figure 8 . Note that we have to exclude $p=\frac{1}{2}$, since condition (A5) is not satisfied in that case.

To use Theorem 4.1, we need to determine the orbits of all the points $a_{n, j}$ and $b_{n, j}$, which in this case are $\frac{1}{3}, \frac{2}{3}$ and 1 . One can easily check that all $\mathrm{KI}_{n}\left(a_{i, j}\right)$ and $\mathrm{KI}_{n}\left(b_{i, j}\right)$ are zero, except for

$$
\mathrm{KI}_{1}\left(\frac{1}{3}\right)=-\frac{1}{6}, \quad \mathrm{KI}_{6}\left(\frac{1}{3}\right)=-\frac{1}{6}, \quad \mathrm{KI}_{6}(1)=1 \quad \text { and } \quad \mathrm{KI}_{4}\left(\frac{2}{3}\right)=-\frac{1}{3} .
$$


The fundamental matrix $M$ of the system is therefore given by

$$
M=\left(\begin{array}{ccccc}
\frac{p-6}{36} & \frac{1-p}{36} & 0 & \frac{p}{12} & \frac{1-p}{12} \\
\frac{1-2 p}{6} & \frac{2 p-1}{6} & 0 & 0 & 0 \\
0 & -\frac{1}{6} & \frac{1}{6} & 0 & 0 \\
\frac{p}{18} & \frac{1-p}{18} & \frac{1}{2} & \frac{p-3}{6} & \frac{1-p}{6} \\
0 & 0 & 0 & \frac{1-2 p}{2} & \frac{2 p-1}{2} \\
\frac{p}{36} & \frac{1-p}{36} & \frac{2}{3} & \frac{p}{12} & -\frac{p+5}{12}
\end{array}\right),
$$

and its null space consists of all vectors of the form

$$
s\left(\begin{array}{lllll}
3 & 3 & 3 & 5 & 5
\end{array}\right)^{\top}, \quad s \in \mathbb{R} .
$$

Again this is a one-dimensional space, so, by Theorem 5.3, $T$ has a unique invariant density. The corresponding measure $m_{\mathbf{p}} \times \mu_{\mathbf{p}}$ is necessarily ergodic for $R$. From

$$
L_{1 / 3}=-\frac{1}{3}, \quad L_{2 / 3}=\frac{2}{3} \cdot \mathbf{1}_{[1 / 3,2 / 3]} \quad \text { and } \quad L_{1}=2
$$

we get the invariant density

$$
h_{\gamma}=\frac{3}{8}\left(3 \cdot \mathbf{1}_{[1 / 3,2 / 3]}+5 \cdot \mathbf{1}_{(2 / 3,1]}\right) .
$$

Let $R:\{0,1\}^{\mathbb{N}} \times\left[\frac{1}{3}, 1\right] \rightarrow\{0,1\}^{\mathbb{N}} \times\left[\frac{1}{3}, 1\right]$ be the pseudo skew-product associated to $T$. For any point $(\omega, x) \in\{0,1\}^{\mathbb{N}} \times\left[\frac{1}{3}, 1\right]$ the frequency of the digit 2 in its random Lüroth expansion is given by

$$
\lim _{n \rightarrow \infty} \frac{1}{n} \sum_{k=0}^{n-1} \mathbf{1}_{\{0,1\}^{\mathbb{N}} \times(1 / 2,1]}\left(R^{k}(\omega, x)\right) .
$$

Since $m_{\mathbf{p}} \times \mu_{\mathbf{p}}$ is ergodic, by the ergodic theorem we have that for $m_{\mathbf{p}} \times \mu_{\mathbf{p}}$-almost every $(\omega, x) \in\{0,1\}^{\mathbb{N}} \times\left[\frac{1}{3}, 1\right]$ the frequency of 2 in the associated random Lüroth expansion is

$$
\int_{(1 / 2,1]} h_{\gamma} d \lambda=\frac{13}{16},
$$

giving also that the frequency of the digit 3 is $3 / 16$.

Even though condition (A5) is not satisfied for $p=\frac{1}{2}$, the fundamental matrix $M$ can still be computed and its null space is still given by $s\left(\begin{array}{l}3 \\ 3\end{array} 355\right)^{\top}, s \in \mathbb{R}$. Moreover, the function $h_{\gamma}=\frac{3}{8}\left(3 \cdot \mathbf{1}_{[1 / 3,2 / 3]}+5 \cdot \mathbf{1}_{(2 / 3,1]}\right)$ is still the unique invariant density. We believe that Theorems 4.1 and 5.3 should still hold without assumption (A5).

Remark 7.1. Note that our method is also capable of handling more general versions of restricted random Lüroth maps. If, instead of considering holes of the form $\left[0, \frac{1}{3}\right)$, we 
restricted the system $\left\{T_{\mathrm{L}}, T_{\mathrm{A}}\right\}$ to an interval $[\eta, 1]$ for some $0<\eta<1$, then by the same arguments as above, the restricted random Lüroth system would have a unique absolutely continuous invariant measure for which the density can be obtained from Theorem 4.1.

Acknowledgement. We would like to thank Karma Dajani for suggesting the use of [Kop90]. The second author is supported by the NWO TOP-Grant No. 614.001.509.

\section{REFERENCES}

[AGH18] N. Abbasi, M. Gharaei and A. J. Homburg. Iterated function systems of logistic maps: synchronization and intermittency. Nonlinearity 31(8) (2018), 3880-3913.

[BBDK94] J. Barrionuevo, R. M. Burton, K. Dajani and C. Kraaikamp. Ergodic properties of generalized Luroth series. TU Delft Report 94-105 (1994), 1-16.

[BG05] W. Bahsoun and P. Góra. Position dependent random maps in one and higher dimensions. Studia Math. 166(3) (2005), 271-286.

[BG97] A. Boyarsky and P. Góra. Laws of Chaos. Invariant Measures and Dynamical Systems in One Dimension (Probability and its Applications). Birkhäuser, Boston, 1997.

[BI09] L. Barreira and G. Iommi. Frequency of digits in the Lüroth expansion. J. Number Theory 129(6) (2009), 1479-1490.

[Buz00] J. Buzzi. Absolutely continuous S.R.B. measures for random Lasota-Yorke maps. Trans. Amer. Math. Soc. 352(7) (2000), 3289-3303.

[DdV05] K. Dajani and M. de Vries. Measures of maximal entropy for random $\beta$-expansions. J. Eur. Math. Soc. 7(1) (2005), 51-68.

[DdV07] K. Dajani and M. de Vries. Invariant densities for random $\beta$-expansions. J. Eur. Math. Soc. 9(1) (2007), 157-176.

[DHK09] K. Dajani, Y. Hartono and C. Kraaikamp. Mixing properties of $(\alpha, \beta)$-expansions. Ergod. Th. \& Dynam. Sys. 29(4) (2009), 1119-1140.

[DK03] K. Dajani and C. Kraaikamp. Random $\beta$-expansions. Ergod. Th. \& Dynam. Sys. 23(2) (2003), 461-479.

[DK10] K. Dajani and C. Kalle. A note on the greedy $\beta$-transformation with arbitrary digits. École de Théorie Ergodique (Séminaires et Congrès, 20). Société Mathématique de France, Paris, 2010, pp. 83-104.

[DK13] K. Dajani and C. Kalle. Local dimensions for the random $\beta$-transformation. New York J. Math. 19 (2013), 285-303.

[EJK90] P. Erdös, I. Joó and V. Komornik. Characterization of the unique expansions $1=\sum_{i=1}^{\infty} q^{-n_{i}}$ and related problems. Bull. Soc. Math. France 118(3) (1990), 377-390.

[EM12] P. Eslami and M. Misiurewicz. Singular limits of absolutely continuous invariant measures for families of transitive maps. J. Difference Equ. Appl. 18(4) (2012), 739-750.

[FLMW10] A. Fan, L. Liao, J. Ma and B. Wang. Dimension of Besicovitch-Eggleston sets in countable symbolic space. Nonlinearity 23(5) (2010), 1185-1197.

[GB03] P. Góra and A. Boyarsky. Absolutely continuous invariant measures for random maps with position dependent probabilities. J. Math. Anal. Appl. 278(1) (2003), 225-242.

[GBI06] P. Góra, A. Boyarsky and S. Islam. Invariant densities of random maps have lower bounds on their supports. J. Appl. Math. Stoch. Anal. 13 (2006), 79175.

[Gel59] A. O. Gel'fond. A common property of number systems. Izv. Akad. Nauk SSSR. Ser. Mat. 23 (1959), 809-814.

[GL16] Y. Gui and W. Li. Hausdorff dimensions of sets related to Lüroth expansion. Acta Math. Hungar. 150(2) (2016), 286-302.

[Gór09] P. Góra. Invariant densities for piecewise linear maps of the unit interval. Ergod. Th. \& Dynam. Sys. 29(5) (2009), 1549-1583.

[Ino12] T. Inoue. Invariant measures for position dependent random maps with continuous random parameters. Studia Math. 208(1) (2012), 11-29.

[JdV69] H. Jager and C. de Vroedt. Lüroth series and their ergodic properties. Indag. Math. 71(1) (1969), $31-42$.

[Kel82] G. Keller. Stochastic stability in some chaotic dynamical systems. Monatsh. Math. 94(4) (1982), 313-333. 
[Kem14] K. Kempton. On the invariant density of the random $\beta$-transformation. Acta Math. Hungar. 142(2) (2014), 403-419.

[KKK90] S. Kalpazidou, A. Knopfmacher and J. Knopfmacher. Lüroth-type alternating series representations for real numbers. Acta Arith. 55(4) (1990), 311-322.

[KKK91] S. Kalpazidou, A. Knopfmacher and J. Knopfmacher. Metric properties of alternating Lüroth series. Port. Math. 48(3) (1991), 319-325.

[Kop90] C. Kopf. Invariant measures for piecewise linear transformations of the interval. Appl. Math. Comput. 39(2, part II) (1990), 123-144.

[LGB+13] Z. Li, P. Góra, A. Boyarsky, H. Proppe and P. Eslami. Family of piecewise expanding maps having singular measure as a limit of ACIMs. Ergod. Th. \& Dynam. Sys. 33(1) (2013), 158-167.

[LM94] A. Lasota and M.C. Mackey. Chaos, Fractals, and Noise. Stochastic Aspects of Dynamics, 2nd edn (Applied Mathematical Sciences, 97). Springer-Verlag, New York, 1994.

[Lür83] J. Lüroth. Ueber eine eindeutige Entwickelung von Zahlen in eine unendliche Reihe. Math. Ann. 21(3) (1883), 411-423.

[LY73] A. Lasota and J. A. Yorke. On the existence of invariant measures for piecewise monotonic transformations. Trans. Amer. Math. Soc. 186 (1973), 1974 481-488.

[LY78] T. Y. Li and J. A. Yorke. Ergodic transformations from an interval into itself. Trans. Amer. Math. Soc. 235 (1978), 183-192.

[Mor85] T. Morita. Random iteration of one-dimensional transformations. Osaka J. Math. 22(3) (1985), 489-518.

[MT13] B. Mance and J. Tseng. Bounded Lüroth expansions: applying Schmidt games where infinite distortion exists. Acta Arith. 158(1) (2013), 33-47.

[Par60] W. Parry. On the $\beta$-expansions of real numbers. Acta Math. Acad. Sci. Hungar. 11 (1960), 401-416.

[Pel84] S. Pelikan. Invariant densities for random maps of the interval. Trans. Amer. Math. Soc. 281(2) (1984), 813-825.

[Rén57] A. Rényi. Representations for real numbers and their ergodic properties. Acta Math. Acad. Sci. Hungar 8 (1957), 477-493.

[Sal68] T. Salát. Zur metrischen Theorie der Lürothschen Entwicklungen der reellen Zahlen. Czechoslovak Math. J. 18 (93) (1968), 489-522.

[SF11] L. Shen and K. Fang. The fractional dimensional theory in Lüroth expansion. Czechoslovak Math. J. 61(136)(3) (2011), 795-807.

[Sid03] N. Sidorov. Almost every number has a continuum of $\beta$-expansions. Amer. Math. Monthly 110(9) (2003), 838-842.

[Suz19] S. Suzuki. Invariant density functions of random $\beta$-transformations. Ergod. Th. \& Dynam. Sys. 39(4) (2019), 1099-1120. 